Pacific

Journal of

Mathematics

QUANTIZATION OF POISSON-HOPF STACKS ASSOCIATED WITH GROUP LIE BIALGEBRAS

Gilles Halbout AND Xiang TANG 


\title{
QUANTIZATION OF POISSON-HOPF STACKS ASSOCIATED WITH GROUP LIE BIALGEBRAS
}

\author{
Gilles Halbout AND Xiang TANG
}

\begin{abstract}
Let $G$ be a simply connected Poisson-Lie group and $\mathfrak{g}$ its Lie bialgebra. Suppose that $\mathfrak{g}$ is a group Lie bialgebra. This means that there is an action of a discrete group $\Gamma$ on $G$ deforming the Poisson structure into coboundary equivalent ones. This induces the existence of a Poisson-Hopf algebra structure on the direct sum over $\Gamma$ of formal functions on $G$, with Poisson structures translated by $\Gamma$. A quantization of this algebra can be obtained by taking the linear dual of a quantization of the $\Gamma$ Lie bialgebra $\mathfrak{g}$, which is the infinitesimal of a $\Gamma$ Poisson-Lie group. In this paper we find out an interesting structure on the dual Lie group $G^{*}$. We prove that we can construct a stack of Poisson-Hopf algebras and prove the existence of the associated deformation quantization of it. This stack can be viewed as the function algebra on "the formal Poisson group" dual to the original $\Gamma$ Poisson-Lie group. To quantize this stack, we apply Drinfeld functors to quantization of the associated $\Gamma$ Lie bialgebra.
\end{abstract}

\section{Introduction}

In this paper, we study examples of Poisson-Hopf stacks and their quantization. Enriquez and Halbout [2008] considered quantization of a $\Gamma$ Lie bialgebra (LBA). As an outcome, they constructed a functor from the category of $\Gamma$ Lie bialgebra to the category of $\Gamma$ quantized universal enveloping algebras (QUE). Our goal here is to study the objects dual to $\Gamma$ Lie bialgebras and their quantizations.

There are two kinds of duality map we can apply to a $\Gamma$ Lie bialgebra: One is to consider the algebra of functions on $G$. We obtain a direct sum $\bigoplus_{\gamma \in \Gamma} \mathcal{O}_{\gamma}$ of formal functions on $G$, with Poisson structures translated by $\Gamma$. When $\Gamma$ is not a finite group, the coproduct $\Delta$ maps $\mathscr{O}_{\gamma}$ to an infinite sum. In general, $\bigoplus_{\gamma \in \Gamma} \mathbb{O}_{\gamma}$ is a Poisson algebra but does not have a Hopf algebra structure because an infinite sum appears in the coproduct. Nevertheless, we will still call $\bigoplus_{\gamma \in \Gamma} \mathrm{O}_{\gamma}$ a $\Gamma$ PoissonHopf algebra. (We do have a collection of Poisson algebras and Poisson morphisms

MSC2000: primary 17B37; secondary 58H05.

Keywords: stack, Poisson, Hopf, Lie bialgebra.

Tang is partially supported by NSF grant 0604552, and thanks Institut de Mathématiques et de Modélisation de Montpellier for its hospitality. 
$\Delta_{\gamma, \gamma^{\prime}}: \mathbb{O}_{\gamma \gamma^{\prime}} \rightarrow \mathbb{O}_{\gamma} \otimes \mathcal{O}_{\gamma^{\prime}}$ that satisfy coassociativity rules). A quantization of such a $\Gamma$ Poisson-Hopf algebra defines the function algebra on a $\Gamma$ quantum group. We refer to [Majid and Sořbel'man 1994] for examples of quantum Weyl groups, and [Enriquez and Halbout 2008] for quantization in the general case.

In this paper, we apply a duality map different from the function dual above. We study the structures on the dual group $G^{*}$ by applying the Drinfeld functor to a $\Gamma$ universal enveloping algebra. We discover a stack of Poisson formal series Hopf algebras (PFSHA as defined in Section 1) dual to a $\Gamma$ Lie bialgebra; this duality is similar to the one between Lie bialgebras and Poisson-Lie groups. Then we study deformation quantization of this stack. We construct the deformation quantization by applying the Drinfeld functor to a $\Gamma$ quantized universal enveloping algebra, and obtain a stack of quantized formal series Hopf algebras (QFSHA). We summarize our results in a commutative diagram:

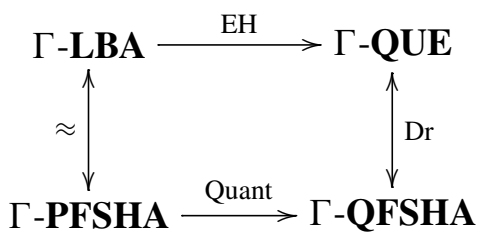

Let $\Gamma$ be a discrete group, $G$ a simply connected Lie group and $\mathfrak{g}$ its Lie algebra. Suppose that $\mathfrak{g}$ is a $\Gamma$ Lie bialgebra (or equivalently that $G$ is a $\Gamma$ Poisson group), that is, a Lie algebra $\left(\mathfrak{g}, \mu_{\mathfrak{g}}\right)$ together with a Lie cobracket $\delta_{e}$, an action of $\Gamma$, $\theta: \Gamma \rightarrow \operatorname{Aut}\left(\mathfrak{g}, \mu_{\mathfrak{g}}\right)$ and $f: \Gamma \rightarrow \bigwedge^{2}(\mathfrak{g})$ a map satisfying compatibility rules such that $\Gamma$ acts on the double. Precise definitions and equivalent categories corresponding to these objects will be recalled in Section 1. Examples of $\Gamma$ Lie bialgebras arise when $G$ is a Poisson-Lie group with Lie bialgebra $\left(\mathfrak{g}, \mu_{\mathfrak{g}}, \delta_{\mathfrak{g}}\right)$, and $\Gamma \subset G$ is a discrete subgroup. Another example is when $\mathfrak{g}$ is a Kac-Moody Lie algebra $\mathfrak{g}$, and $\Gamma$ is a covering of the Weyl group of $\mathfrak{g}$. In the latter case, a quantization was given [Majid and Sorbel'man 1994]. Quantization of a general $\Gamma$ Lie bialgebra was done in [Enriquez and Halbout 2008], as we will review in Section 1.

What structure does one get on the corresponding dual groups? Considering the function algebra of a formal group, we get a trivial stack of Poisson-Hopf algebras. In Section 3, we prove that we get a nontrivial stack of Poisson algebras of functions on the formal Poisson-Lie group $G^{*}$ dual to a $\Gamma$ Poisson-Lie group $G$. To do so, we will construct "lifts" of the elements $(f(\gamma))_{\gamma \in \Gamma}$ in the function algebra on $G^{*}$. In Section 2, we recall basic definitions of stacks and explain our main results.

In Section 4, we construct quantization of these nontrivial Poisson-Hopf stacks. To do so we use quantization [Enriquez and Halbout 2008] of a $\Gamma$ Lie bialgebra. To deduce from it a quantization of a nontrivial Poisson-Hopf stack, we use the 
Drinfeld functor and prove that quantization of the elements $(f(\gamma))_{\gamma \in \Gamma}$ can be made "admissible", that is, they will give quantizations of the corresponding "lifts".

Finally, in Section 5, we give an explicit example corresponding to the case where $G$ is a simple Lie group and $\Gamma$ is a covering of the corresponding Weyl group. In this case, quantization of Majid and Soíbel'man [1994] will lead to an explicit quantization of the nontrivial Poisson-Hopf stack.

Our results fit very well within Bressler, Gorokhovsky, Nest and Tsygan's framework [Bressler et al. 2007] of deformation quantization of gerbes. On one hand, our results provide interesting examples of quantization of stacks; on the other, the problems we deal with in this paper are more special and complicated because we need to treat Hopf algebra structure. In [Kirillov and Reshetikhin 1990] and [Soľbel'man 1991] quantum Weyl groups are used to study R-matrices, and we hope that the results in this paper will shed a light on the general $\Gamma$ R-matrices.

\section{1. $\Gamma$ Lie bialgebras and equivalent categories}

We recall some results of [Enriquez and Halbout 2008].

$\Gamma$ Lie algebras. A group Lie algebra is a triple $\left(\Gamma, \mathfrak{g}, \theta_{\mathfrak{g}}\right)$, where $\Gamma$ is a group, $\mathfrak{g}$ is a Lie algebra and $\theta_{\mathfrak{g}}: \Gamma \rightarrow \operatorname{Aut}(\mathfrak{g})$ is a group morphism. It is the infinitesimal version of a $\Gamma$ action on a group $G$. Group Lie algebras form a category.

If $\Gamma$ is a discrete group, a $\Gamma$ Lie algebra is a pair $\left(\mathfrak{g}, \theta_{\mathfrak{g}}\right)$ such that $\left(\Gamma, \mathfrak{g}, \theta_{\mathfrak{g}}\right)$ is a group Lie algebra. $\Gamma$ Lie algebras form a subcategory of group Lie algebras. Such a $\Gamma$ Lie algebra will be said to be the infinitesimal of a $\Gamma$ group $G$.

A group cocommutative bialgebra is a triple $(\Gamma, U, i)$, where $\Gamma$ is a group, $U$ is a cocommutative bialgebra, $U=\bigoplus_{\gamma \in \Gamma} U_{\gamma}$ is a decomposition of $U$ and $i: \mathbf{k} \Gamma \rightarrow U$ is a bialgebra morphism, such that $U_{\gamma} U_{\gamma^{\prime}} \subset U_{\gamma \gamma^{\prime}}, \Delta_{U}\left(U_{\gamma}\right) \subset U_{\gamma}^{\otimes 2}$ and $i$ is compatible with the $\Gamma$ grading.

We then define a $\Gamma$ cocommutative bialgebra as a pair $(U, i)$ such that $(\Gamma, U, i)$ is a group cocommutative bialgebra. $\Gamma$ cocommutative bialgebras form a category.

The category of group (or $\Gamma$ ) cocommutative bialgebras contains as a full subcategory the category of group (respectively $\Gamma$ ) universal enveloping algebras, where $(U, \Gamma, i)$ satisfies the additional requirement that $U_{e}$ is a universal enveloping algebra.

Let 0 be a commutative algebra (in a symmetric monoidal category $\mathscr{S}$ ) with a decomposition $\mathbb{O}=\bigoplus_{\gamma \in \Gamma} \mathbb{O}_{\gamma}$. Suppose that $\mathbb{O}_{\gamma} \mathbb{O}_{\gamma^{\prime}}=0$ for $\gamma \neq \gamma^{\prime}$ and that we have algebra morphisms

$$
\Delta_{\gamma^{\prime} \gamma^{\prime \prime}}: \mathbb{O}_{\gamma^{\prime} \gamma^{\prime \prime}} \rightarrow \mathscr{O}_{\gamma^{\prime}} \otimes \mathcal{O}_{\gamma^{\prime \prime}}, \quad \eta: \mathbf{k} \rightarrow \mathscr{O}_{e}, \quad \varepsilon: \mathbb{O}_{e} \rightarrow \mathbf{k}
$$

satisfying axioms such that these morphisms add up to a bialgebra structure on 0 when $\Gamma$ is finite. Then we define a group commutative bialgebra (in a symmetric 
monoidal category $\mathscr{Y})$ as a triple $(\Gamma, \mathcal{O}, j)$, where $\Gamma$ is a group and $j: \mathcal{O} \rightarrow \mathbf{k}^{\Gamma}$ is a morphism of commutative algebras compatible with the $\Gamma$ gradings and the maps $\Delta_{\gamma^{\prime} \gamma^{\prime \prime}}$ on both sides. We define $\Gamma$ commutative bialgebras as above.

Define the category of group (or $\Gamma$ ) formal series Hopf (FSH) algebras as a full subcategory of the category of group (respectively $\Gamma$ ) commutative bialgebras in $\mathscr{Y}=$ \{provector spaces\} by the condition that $\mathrm{O}_{e}$ (or equivalently, each $\mathrm{O}_{\gamma}$ ) is a formal series algebra. Such an FSH algebra corresponds to functions on the formal dual group of a $\Gamma$ group $G$.

Proposition 1.1 [Enriquez and Halbout 2008]. (1) We have (anti)equivalences of categories

$$
\begin{aligned}
\{\text { group Lie algebras }\} & \leftrightarrow\{\text { group universal enveloping algebras }\} \\
& \leftrightarrow\{\text { group FHS algebras }\}
\end{aligned}
$$

where the last map is an antiequivalence.

(2) If $\Gamma$ is a group, these (anti)equivalences restrict to

$\{\Gamma$-Lie algebras $\} \leftrightarrow\{\Gamma$-universal enveloping algebras $\} \leftrightarrow\{\Gamma$-FHS algebras $\}$.

We denote the $\Gamma$ universal enveloping algebra corresponding to a $\Gamma$ Lie algebra $\left(\Gamma, \mathfrak{g}, \theta_{\mathfrak{g}}\right)$ as $U(\mathfrak{g}) \rtimes \Gamma$. It is isomorphic to $U(\mathfrak{g}) \otimes \mathbf{k} \Gamma$ as a vector space. If we denote by $x \mapsto[x]$ and $\gamma \mapsto[\gamma]$ the natural maps $\mathfrak{g} \rightarrow U(\mathfrak{g}) \rtimes \Gamma$ and $\Gamma \rightarrow U(\mathfrak{g}) \rtimes \Gamma$, then the bialgebra structure of $U(\mathfrak{g}) \rtimes \Gamma$ is given by

$$
\begin{aligned}
& {[\gamma][x]\left[\gamma^{-1}\right]=\left[\theta_{\gamma}(x)\right], \quad[\gamma]\left[\gamma^{\prime}\right]=\left[\gamma \gamma^{\prime}\right], \quad \Delta([\gamma])=[\gamma] \otimes[\gamma],} \\
& {[x]\left[x^{\prime}\right]-\left[x^{\prime}\right][x]=\llbracket x, x^{\prime} \rrbracket, \quad[e]=1, \quad \Delta([x])=[x] \otimes 1+1 \otimes[x] .}
\end{aligned}
$$

When $\Gamma$ is finite, the corresponding $\Gamma$ FSH algebra is then $(U(\mathfrak{g}) \rtimes \mathbf{k} \Gamma)^{*}$, and in general, this is $\bigoplus_{\gamma \in \Gamma}(U(\mathfrak{g}) \otimes \mathbf{k} \gamma)^{*}$.

\section{Г Lie bialgebras.}

Definition 1.2. A group Lie bialgebra is a 5-uple $\left(\Gamma, \mathfrak{g}, \theta_{\mathfrak{g}}, \delta_{\mathfrak{g}}, f\right)$, where $\left(\Gamma, \mathfrak{g}, \theta_{\mathfrak{g}}\right)$ is a group Lie algebra, $\delta_{\mathfrak{g}}: \mathfrak{g} \rightarrow \bigwedge^{2}(\mathfrak{g})$ is ${ }^{1}$ such that $\left(\mathfrak{g}, \delta_{\mathfrak{g}}\right)$ is a Lie bialgebra, and $f: \Gamma \rightarrow \bigwedge^{2}(\mathfrak{g})$ is a map $\gamma \mapsto f_{\gamma}$ such that

(a) $\bigwedge^{2}\left(\theta_{\gamma}\right) \circ \delta \circ \theta_{\gamma}^{-1}(x)=\delta(x)+\left[f_{\gamma}, x \otimes 1+1 \otimes x\right]$ for any $x \in \mathfrak{g}$,

(b) $f_{\gamma \gamma^{\prime}}=f_{\gamma}+\bigwedge^{2}\left(\theta_{\gamma}\right)\left(f_{\gamma^{\prime}}\right)$,

(c) $(\delta \otimes \mathrm{id})\left(f_{\gamma}\right)+\left[f_{\gamma}^{1,3}, f_{\gamma}^{2,3}\right]+$ cyclic permutations $=0$.

\footnotetext{
${ }^{1}$ We view $\bigwedge^{2}(V)$ as a subspace of $V^{\otimes 2}$.
} 
Group Lie bialgebras form a category. When $\Gamma$ is fixed, one defines the category of $\Gamma$ Lie bialgebras as above.

A co-Poisson structure on a group cocommutative bialgebra $(\Gamma, U, i)$ is a coPoisson structure $\delta_{U}: A \rightarrow \bigwedge^{2}(U)$ such that $\delta_{U}\left(U_{\gamma}\right) \subset \bigwedge^{2}\left(U_{\gamma}\right)$. Co-Poisson group cocommutative bialgebras form a category.

Co-Poisson group universal enveloping algebras form a full subcategory of the latter category. One defines the full subcategories of co-Poisson $\Gamma$ cocommutative bialgebras and co-Poisson $\Gamma$ enveloping algebras as above.

A Poisson structure on a group commutative bialgebra $(\Gamma, \mathcal{O}, j)$ is a Poisson bialgebra structure $\{\cdot, \cdot\}: \bigwedge^{2}(\mathbb{O}) \rightarrow \mathcal{O}$ such that $\left\{O_{\gamma}, \mathscr{O}_{\gamma}\right\} \subset \mathbb{O}_{\gamma}$ and $\left\{\mathcal{O}_{\gamma}, \mathscr{O}_{\gamma^{\prime}}\right\}=0$ if $\gamma \neq \gamma^{\prime}$. Poisson group bialgebras form a category, and Poisson group FSH algebras form a full subcategory when $\mathscr{S}=$ \{provector spaces\}. One defines the full subcategories of Poisson $\Gamma$ bialgebras and Poisson $\Gamma$ FSH algebras as above.

Example. Let $G$ be a Poisson-Lie (for example, algebraic) group, and let $\Gamma \subset G$ be a subgroup (which we view as an abstract group). We define $\theta_{\gamma}:=\operatorname{Ad}(\gamma)$, where Ad: $G \rightarrow \operatorname{Aut}_{\text {Lie }}(\mathfrak{g})$ is the adjoint action. If $P: G \rightarrow \bigwedge^{2}(\mathfrak{g})$ is the Poisson bivector satisfying $P\left(g g^{\prime}\right)=P\left(g^{\prime}\right)+\bigwedge^{2}\left(\operatorname{Ad}\left(g^{\prime}\right)\right)(P(g))$, then we set $f_{\gamma}:=-P(\gamma)$. Then $(\mathfrak{g}, \Gamma, f)$ is a $\Gamma$ Lie bialgebra.

Example. Let $\left(\mathfrak{g}, r_{\mathfrak{g}}\right)$ be a quasitriangular Lie bialgebra and let $\theta: \Gamma \rightarrow \operatorname{Aut}\left(\mathfrak{g}, t_{\mathfrak{g}}\right)$ be an action of $\Gamma$ on $\mathfrak{g}$ by Lie algebra automorphisms preserving $t_{\mathfrak{g}}:=r_{\mathfrak{g}}+r_{\mathfrak{g}}^{2,1}$. If we set $f_{\gamma}:=\theta_{\gamma}^{\otimes 2}(r)-r$, then $(\mathfrak{g}, \theta, f)$ is a $\Gamma$ Lie bialgebra (we call this a quasitriangular $\Gamma$ Lie bialgebra). For example, $\mathfrak{g}$ is a Kac-Moody Lie algebra, and $\Gamma=\widetilde{W}$ is a covering of the Weyl group of $\mathfrak{g}$; see [Majid and Sorbel'man 1994].

Proposition 1.3 [Enriquez and Halbout 2008]. (1) We have category (anti)equivalences

$\{$ group bialgebras $\} \leftrightarrow\{$ co-Poisson group universal enveloping algebras $\}$ $\leftrightarrow\{$ Poisson group FSH algebras $\}$.

(2) The (anti)equivalences above restrict to category (anti)equivalences $\{\Gamma$-bialgebras $\} \leftrightarrow\{$ co-Poisson $\Gamma$ universal enveloping algebras $\}$ $\leftrightarrow\{$ Poisson $\Gamma$ FSH algebras $\}$.

If $\left(\mathfrak{g}, \theta_{\mathfrak{g}}, \delta_{\mathfrak{g}}\right)$ is a $\Gamma$ Lie bialgebra, then the co-Poisson structure on $U:=U(\mathfrak{g}) \rtimes \Gamma$ is given by $\delta_{U}([x])=\left[\delta_{\mathfrak{g}}(x)\right]$ and $\delta_{U}([\gamma])=-\left[f_{\gamma}\right]([\gamma] \otimes[\gamma])$. Here we also denote by $x \mapsto[x]$ the natural map $\bigwedge^{2}(\mathfrak{g}) \rightarrow \bigwedge^{2}(U(\mathfrak{g}) \rtimes \Gamma)$.

Quantization of $\Gamma$ Lie bialgebras. In a symmetric monoidal category $\mathscr{T}$, let a $\Gamma$ graded bialgebra be a bialgebra $A$ (in $\mathscr{S}$ ) equipped with a grading $A=\bigoplus_{\gamma \in \Gamma} A_{\gamma}$, such that $A_{\gamma} A_{\gamma^{\prime}} \subset A_{\gamma \gamma^{\prime}}$ and $\Delta_{A}\left(A_{\gamma}\right) \subset A_{\gamma}^{\otimes 2}$. 
Assume that $A$ is a $\Gamma$ graded bialgebra in the category of topologically free $\mathbf{k} \llbracket \hbar \rrbracket$-modules and that $A$ is quasicocommutative in that $A_{0}:=A / \hbar A$ is cocommutative. Then we get a co-Poisson structure on $A_{0}$. It is $\Gamma$ graded in that $\delta_{A_{0}}\left(\left(A_{0}\right)_{\gamma}\right) \subset \Lambda^{2}\left(\left(A_{0}\right)_{\gamma}\right)$. We therefore get a classical limit functor, class, from $\{\Gamma$-graded quasicocommutative bialgebras $\}$ to $\{\Gamma$-graded co-Poisson bialgebras $\}$.

Definition 1.4. A quantization functor for $\Gamma$ Lie bialgebras is a functor \{co-Poisson $\Gamma$ universal enveloping algebras\}

$\rightarrow\{\Gamma$-graded quasicocommutative bialgebras $\}$,

which is right inverse to class.

Assume that $(\mathfrak{g}, \theta, f)$ is a $\Gamma$ Lie bialgebra. Let $\left(U_{e}, *, \Delta_{e}\right)$ be the EtingofKazhdan quantization of $(\mathfrak{g}, \delta)$; we will denote the multiplication by $m_{e}$. We get this from [Enriquez and Halbout 2008]:

Proposition 1.5. There exist sets $\left(\mathrm{F}_{\gamma, \gamma \gamma^{\prime}}\right)_{\gamma, \gamma^{\prime} \in \Gamma}$ of elements in $U^{\otimes 2}$, with $\mathrm{F}_{\gamma, \gamma \gamma^{\prime}}=$ $1+\hbar \mathrm{f}_{1}+O\left(\hbar^{2}\right)$ and $\operatorname{Alt}\left(\mathrm{f}_{1}\right)=\bigwedge^{2}\left(\theta_{\gamma}\right)\left(f_{\gamma^{\prime}}\right)$, sets $\left(\mathrm{v}_{\gamma, \gamma \gamma^{\prime}, \gamma \gamma^{\prime} \gamma^{\prime \prime}}\right)_{\gamma, \gamma^{\prime}, \gamma^{\prime \prime} \in \Gamma \text { of elements }}$ in $1+\hbar^{2} U$, sets $\left(U_{\gamma}, m_{\gamma}, \Delta_{\gamma}\right)_{\gamma \in \Gamma}$ of bialgebras, and sets $\left(\mathrm{i}_{\gamma, \gamma \gamma^{\prime}}\right)_{\gamma, \gamma^{\prime} \in \Gamma}$ of algebra morphisms from $\left(U_{\gamma}, m_{\gamma}\right)$ to $\left(U_{\gamma \gamma^{\prime}}, m_{\gamma \gamma^{\prime}}\right)$, such that

- $\Delta_{\gamma}=\mathrm{i}_{e, \gamma}^{\otimes 2} \circ \operatorname{Ad}\left(\mathrm{F}_{e, \gamma}\right) \circ \Delta_{e} \circ \mathrm{i}_{e, \gamma}^{-1}$,

- $\left(\mathrm{F}_{e, \gamma} \otimes 1\right) *\left(\Delta_{e} \otimes \mathrm{id}\right)\left(\mathrm{F}_{e, \gamma}\right)=\left(1 \otimes \mathrm{F}_{e, \gamma}\right) *\left(\mathrm{id} \otimes \Delta_{e}\right)\left(\mathrm{F}_{e, \gamma}\right)$,

- $\mathrm{F}_{e, \gamma \gamma^{\prime}}=\mathrm{v}_{e, \gamma, \gamma \gamma^{\prime}}^{\otimes 2} *\left(\mathrm{i}_{e, \gamma}^{\otimes 2}\right)^{-1}\left(\mathrm{~F}_{\gamma, \gamma \gamma^{\prime}}\right) * \mathrm{~F}_{e, \gamma} * \Delta_{e}\left(\mathrm{v}_{e, \gamma, \gamma \gamma^{\prime}}\right)^{-1}$,

- $\mathrm{i}_{e, \gamma \gamma^{\prime}}=\mathrm{i}_{\gamma, \gamma \gamma^{\prime}} \circ \mathrm{i}_{e, \gamma} \circ \operatorname{Ad}\left(\mathrm{v}_{e, \gamma, \gamma \gamma^{\prime}}^{-1}\right)$,

- $\mathrm{v}_{e, \gamma \gamma^{\prime}, \gamma \gamma^{\prime} \gamma^{\prime \prime}} * \mathrm{v}_{e, \gamma, \gamma \gamma^{\prime}}=\mathrm{v}_{e, \gamma, \gamma \gamma^{\prime} \gamma^{\prime \prime}} * \mathrm{i}_{e, \gamma}^{-1}\left(\mathrm{v}_{\gamma, \gamma \gamma^{\prime}, \gamma \gamma^{\prime} \gamma^{\prime \prime}}\right)$.

Here to make the formulas shorter we have chosen to write the above equations with e being the unit of the group $\Gamma$; however, the formulas are still valid if we replace $e$ by any other element of the group and multiply $\gamma, \gamma \gamma^{\prime}$ and $\gamma \gamma^{\prime} \gamma^{\prime \prime}$ on the left by this element.

We then get a quantization of the $\Gamma$ Lie bialgebra by setting $U=S(\mathfrak{g}) \otimes \mathbf{k} \Gamma \llbracket \hbar \rrbracket$ and putting $[x \mid \gamma]:=x \otimes \gamma$ and $\left[x \otimes x^{\prime} \mid \gamma, \gamma^{\prime}\right]:=(x \otimes \gamma) \otimes\left(x^{\prime} \otimes \gamma^{\prime}\right) \in U^{\otimes 2}$.

There are unique linear maps $m: U^{\otimes 2} \rightarrow U$ and $\Delta: U \rightarrow U^{\otimes 2}$ such that

$$
\begin{aligned}
m:[x \mid \gamma]\left[x^{\prime} \mid \gamma^{\prime}\right] & \mapsto\left[x * \mathrm{i}_{e, \gamma}^{-1}\left(\theta_{\gamma}\left(x^{\prime}\right)\right) * \mathrm{v}_{e, \gamma, \gamma \gamma^{\prime}}^{-1} \mid \gamma \gamma^{\prime}\right], \\
\Delta:[x \mid \gamma] & \mapsto\left[\Delta_{e}(x) * \mathrm{~F}_{e, \gamma}^{-1} \mid \gamma, \gamma\right] .
\end{aligned}
$$

The unit for $U$ is $[1 \mid e]$, and the counit is the map $[x \mid \gamma] \mapsto \delta_{\gamma, e} \varepsilon(x)$.

Proposition 1.6 [Enriquez and Halbout 2008]. This defines a bialgebra structure on $U$, quantizing the co-Poisson bialgebra structure induced by $(\mathfrak{g}, \theta, f)$. 


\section{Stacks and main results}

It is well known that the semiclassical limit of a quantum group is a PoissonLie group. In this paper, we attempt to answer, What is the semiclassical limit corresponding to a " $\Gamma$ coboundary quantum group"? We hope to say that this semiclassical object is a "stack" of Poisson-Lie groups $G^{*}$ over the classifying stack $B \Gamma$ of the group $\Gamma$. Toward this, we construct a stack of Poisson-Hopf algebras over the groupoid $\Gamma \rtimes \Gamma$ (a transformation groupoid associated to the $\Gamma$ right action on $\Gamma$ ) and also a quantization of such a stack. Because of the existence of the twisted cocycle, we expect that such an algebroid stack is not trivial. We also hope that our construction will eventually lead to a complete description of the semiclassical limit of a $\Gamma$-coboundary quantum group.

Definition 2.1. A stack on $M$ is

- an open cover of $M=\bigcup U_{i}$,

- a sheaf of rings $A_{i}$ on every $U_{i}$,

- an isomorphism of sheaves of rings $G_{i j}:\left.\left.A_{j}\right|_{U_{i} \cap U_{j}} \rightarrow A_{i}\right|_{U_{i} \cap U_{j}}$ for every $i, j$,

- an invertible element $\left.c_{i j k} \in A_{i}\right|_{U_{i} \cap U_{j} \cap U_{k}}$ for every $i, j, k$ satisfying

(1) $G_{i j} G_{j k}=\operatorname{Ad}\left(c_{i j k}\right) G_{i k}$ and

(2) $c_{i j k} c_{i k l}=G_{i j}\left(c_{j k l}\right) c_{i j l}$ for every $i, j, k, l$.

If two such data $\left(U_{i}^{\prime}, A_{i}^{\prime}, G_{i j}^{\prime}, c_{i j k}^{\prime}\right)$ and $\left(U_{i}^{\prime \prime}, A_{i}^{\prime \prime}, G_{i j}^{\prime \prime}, c_{i j k}^{\prime \prime}\right)$ are given on $M$, an isomorphism between them is

- an open cover $M=\bigcup U_{i}$ refining both $\left\{U_{i}^{\prime}\right\}$ and $\left\{U_{i}^{\prime \prime}\right\}$,

- isomorphisms $H_{i}: A_{i}^{\prime} \rightarrow A_{i}^{\prime \prime}$ on $U_{i}$, and

- invertible elements $b_{i j}$ of $\left.A_{i}^{\prime}\right|_{U_{i} \cap U_{j}}$ such that

(1) $G_{i j}^{\prime \prime}=H_{i} \operatorname{Ad}\left(b_{i j}\right) G_{i j}^{\prime} H_{j}^{-1}$ and

(2) $H_{i}^{-1}\left(c_{i j k}^{\prime \prime}\right)=b_{i j} G_{i j}^{\prime}\left(b_{j k}\right) c_{i j k} b_{i k}^{-1}$.

Inspired by Definition 2.1, we define a stack over a discrete groupoid. Let $\mathfrak{G}$ be a discrete groupoid with its unit space $\mathfrak{G}_{0}$.

Definition 2.2. A stack on $\mathfrak{G}$ consists of

- a collection of rings $A_{x}$ on every point $x$ of $\mathfrak{G}_{0}$,

- an isomorphism of sheaves of rings $T_{g}: A_{t(g)} \rightarrow A_{s(g)}$ for every arrow $g \in \mathfrak{G}$, where $s, t$ are the source and target maps of $\mathfrak{G}$, and

- an invertible element $c_{g_{1}, g_{2}} \in A_{s\left(g_{1}\right)}$ for every pair of composable arrows in $\mathfrak{G}$ such that

(1) $T_{g_{1}} \circ T_{g_{2}}=\operatorname{Ad}\left(c_{g_{1}, g_{2}}\right) T_{g_{1} g_{2}}$, where by $\operatorname{Ad}\left(c_{g_{1}, g_{2}}\right)$ we mean the conjugation operator on $A_{s\left(g_{1}\right)}$ associated to the invertible element $c_{g_{1}, g_{2}}$, and 
(2) $c_{g_{1}, g_{2}} c_{g_{1} g_{2}, g_{3}}=T_{g_{1}}\left(c_{g_{2}, g_{3}}\right) c_{g_{1}, g_{2} g_{3}}$ for every triple of composable arrows $g_{1}, g_{2}, g_{3}$ in $\mathfrak{G}$.

One can generalize the equivalence between stacks of Definition 2.1, but we omit the details here.

Our main result will involve the notion of a stack of Poisson-Hopf algebras.

Definition 2.3. A stack of Poisson-Hopf algebras over a discrete groupoid $\mathfrak{G}$ is

- a collection $A_{x}$ of Poisson-Hopf algebras $\left(A_{x}, m_{x}, \Delta_{x},\{\cdot, \cdot\}_{x}\right)_{x \in \mathfrak{G}_{0}}$,

- a Poisson morphism $T_{g}: A_{t(g)} \rightarrow A_{s(g)}$ for $g \in \mathfrak{G}$, and

- an invertible element $c_{g_{1}, g_{2}} \in A_{s\left(g_{1}\right)}$ for every pair of composable arrows in $\mathfrak{G}$ such that

(1) $T_{g_{1}} \circ T_{g_{2}}=\operatorname{Ad}\left(c_{g_{1}, g_{2}}\right) T_{g_{1} g_{2}}$, where by $\operatorname{Ad}\left(c_{g_{1}, g_{2}}\right)$, we mean the conjugation operator on $A_{s\left(g_{1}\right)}$ associated to the invertible element $c_{g_{1}, g_{2}}$, and

(2) $c_{g_{1}, g_{2}} c_{g_{1} g_{2}, g_{3}}=T_{g_{1}}\left(c_{g_{2}, g_{3}}\right) c_{g_{1}, g_{2} g_{3}}$ for every triple of composable arrows $g_{1}, g_{2}, g_{3}$.

In what follows, we consider the groupoid $\Gamma \rtimes \Gamma$ defined by the action of $\Gamma$ on $\Gamma$ itself by right multiplication. As $\Gamma$ is discrete, $\Gamma \rtimes \Gamma$ is a discrete groupoid. We will use $\left(\gamma, \gamma \gamma^{\prime}\right)$ to denote an arrow in $\Gamma \rtimes \Gamma$ mapping from $\gamma$ to $\gamma \gamma^{\prime}$. The product of a pair of composable arrows $\left(\gamma, \gamma \gamma^{\prime}\right)$ and $\left(\gamma \gamma^{\prime}, \gamma \gamma^{\prime} \gamma^{\prime \prime}\right)$ in $\Gamma \rtimes \Gamma$ is $\left(\gamma, \gamma \gamma^{\prime} \gamma^{\prime \prime}\right)$. For our main results, we associate an Poisson-Hopf algebra $\mathbb{O}_{G_{\gamma}^{*}}$ to each point $\gamma$ in the unit space of $\Gamma \rtimes \Gamma$, and we will prove the existence of a stack of Poisson-Hopf algebras over the groupoid $\Gamma \rtimes \Gamma$.

Theorem 2.4. Associated to a coboundary Lie bialgebra $\left(\Gamma, \mathfrak{g}, \theta_{\mathfrak{g}}, \delta_{\mathfrak{g}}, f\right)$, there is a stack of Poisson-Hopf algebras over the groupoid $\Gamma \rtimes \Gamma$.

To be compatible with the result of Proposition 1.5, we will mainly prove the following results.

- There is a set $\left(\mathcal{O}_{G_{\gamma}^{*}}\right)_{\gamma \in \Gamma}$ of Poisson-Hopf algebras $\left(\mathcal{O}_{G_{\gamma}^{*}}, m_{\gamma}, \Delta_{\gamma},\{\cdot, \cdot\}_{\gamma}\right)_{\gamma \in \Gamma}$.

- Associated to each arrow $\left(\gamma, \gamma \gamma^{\prime}\right)$ in $\Gamma \rtimes \Gamma$, there is a Poisson morphism $j_{\gamma, \gamma \gamma^{\prime}}$ from $O_{G_{\gamma}^{*}}$ to $O_{G_{\gamma \gamma^{*}}^{*}}$.

- Associated to a pair of composable arrows $\left(\gamma, \gamma \gamma^{\prime}\right)$ and $\left(\gamma \gamma^{\prime}, \gamma \gamma^{\prime} \gamma^{\prime \prime}\right)$ in $\Gamma \rtimes \Gamma$, there is an element $u_{\gamma, \gamma \gamma^{\prime}, \gamma \gamma^{\prime} \gamma^{\prime \prime}}$ of $O_{G_{\gamma}^{*}}$ satisfying relations

(1) $j_{\gamma, \gamma \gamma^{\prime} \gamma^{\prime \prime}}=j_{\gamma \gamma^{\prime}, \gamma \gamma^{\prime} \gamma^{\prime \prime}} \circ j_{\gamma, \gamma \gamma^{\prime}} \circ \operatorname{Ad}_{\star_{\gamma}}\left(u_{\gamma, \gamma \gamma^{\prime}, \gamma \gamma^{\prime} \underline{\gamma}_{-}^{\prime \prime}}^{-1}\right)$, where $\operatorname{Ad}_{\star_{\gamma}}\left(u_{\gamma, \gamma \gamma^{\prime}, \gamma \gamma^{\prime} \gamma^{\prime \prime}}^{-1}\right)$ is the conjugation operator associated to $u_{\gamma, \gamma \gamma^{\prime}, \gamma \gamma^{\prime} \gamma^{\prime \prime}}^{-1}$ with respect to the Baker-Campbell-Hausdorff product $\star_{\gamma}$, and

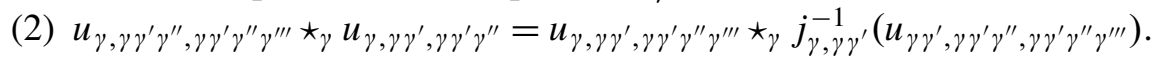


With the above data $\left(\left(\mathcal{O}_{G_{\gamma}^{*}}, m_{\gamma}, \Delta_{\gamma},\{\cdot, \cdot\}_{\gamma}\right)_{\gamma \in \Gamma}, j_{\gamma, \gamma \gamma^{\prime}}, u_{\gamma, \gamma \gamma^{\prime}, \gamma \gamma^{\prime} \gamma^{\prime \prime}}\right)$, if we define $T_{\gamma, \gamma \gamma^{\prime}}: \mathscr{O}_{G_{\gamma \gamma^{\prime}}^{*}} \rightarrow \mathscr{O}_{G_{\gamma}^{*}}$ by $T_{\gamma, \gamma \gamma^{\prime}}=j_{\gamma, \gamma \gamma^{\prime}}^{-1}$ and $c_{\left(\gamma, \gamma \gamma^{\prime}\right),\left(\gamma \gamma^{\prime}, \gamma \gamma^{\prime} \gamma^{\prime \prime}\right)} \in \mathscr{O}_{G_{\gamma}^{*}}$ to be $u_{\gamma, \gamma \gamma^{\prime}, \gamma \gamma^{\prime} \gamma^{\prime \prime}}^{-1}$, then we can easily check that we do obtain a stack of Poisson-Hopf algebras over $\Gamma \rtimes \Gamma$ satisfying Definition 2.2.

We will then prove the existence of a stack of Hopf algebras quantizing this stack of Poisson-Hopf algebras:

Theorem 2.5. There is a stack of Hopf algebras quantizing the stack of PoissonHopf algebras over $\Gamma \rtimes \Gamma$ defined in Theorem 2.4. Namely, we have a collection of the following data: ${ }^{2}$

- $\left(\mathbb{A}_{\gamma}, *_{\gamma}\right)_{\gamma \in \Gamma}$, which are deformation quantizations ${ }^{3}$ of the Poisson algebras $\left(\mathrm{O}_{G_{\gamma}^{*}},\{\cdot, \cdot\}_{\gamma}\right)_{\gamma \in \Gamma}$,

- algebra morphisms $i_{\gamma, \gamma \gamma^{\prime}}: \mathbb{A}_{\gamma} \rightarrow \mathbb{A}_{\gamma \gamma^{\prime}}$, and

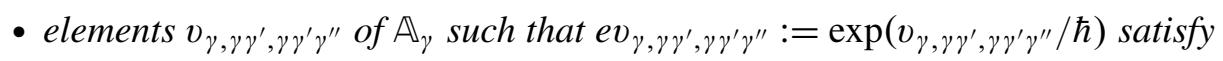
relations

(1) $i_{\gamma, \gamma \gamma^{\prime} \gamma^{\prime \prime}}=i_{\gamma \gamma^{\prime}, \gamma \gamma^{\prime} \gamma^{\prime \prime}} \circ i_{\gamma, \gamma \gamma^{\prime}} \circ \operatorname{Ad}\left(e v_{\gamma, \gamma \gamma^{\prime}, \gamma \gamma^{\prime} \gamma^{\prime \prime}}^{-1}\right)$ and

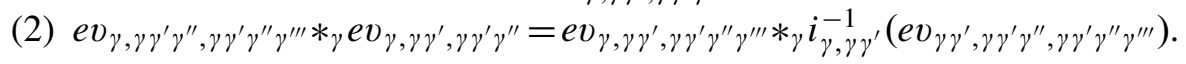

From our setup in this section, one can see that the facts that $\Gamma$ is a group and that we have a transformation groupoid $\Gamma \rtimes \Gamma$ are not crucial in our construction. It is natural to expect a more general theory for quantization of a $\mathfrak{G}$-coboundary Lie bialgebras with $\mathfrak{G}$ a discrete groupoid or even just category.

\section{A stack of Poisson bialgebras of functions on the formal group $G^{*}$}

Let $\left(\mathfrak{g}, \theta_{\mathfrak{g}}, \delta_{\mathfrak{g}}, f\right)$ be a $\Gamma$ Lie bialgebra. In this section we will construct a stack of Poisson bialgebras of functions on a formal Poisson group $G^{*}$.

Notations. Let $(\mathfrak{g}, \delta)$ be a Lie bialgebra. Let $\left(U(\mathfrak{g}), \Delta_{0}, \delta\right)$ be its corresponding cocommutative co-Poisson bialgebra, which can be seen as the dual of the function algebra of the formal Poisson-Lie group $G$ corresponding to $(\mathfrak{g}, \delta)$. In the same way, we will define $\mathscr{O}_{G^{*}}$ as the commutative Poisson-Hopf algebra of functions of the formal Poisson-Lie group $G^{*}$ corresponding to the dual Lie bialgebra $\mathfrak{g}^{*}$. We define by $\mathfrak{m}_{G^{*}} \subset \mathcal{O}_{G^{*}}$ the maximal ideal of this ring. If $k$ is an integer $\geq 1$, we denote by $\mathcal{O}_{\left(G^{*}\right)^{k}}$ the ring of formal functions on $\left(G^{*}\right)^{k}$, by $\mathfrak{m}_{\left(G^{*}\right)^{k}}$ its maximal ideal, and by $\mathfrak{m}_{\left(G^{*}\right)^{k}}^{i}$ the $i$-th power of this ideal.

If $f, g \in \mathfrak{m}_{\left(G^{*}\right)^{k}}^{2}$, then the series

$$
f \star g=f+g+\frac{1}{2}\{f, g\}+\cdots+B_{n}(f, g)+\cdots
$$

\footnotetext{
${ }^{2}$ Similarly to what we did for Theorem 2.4, we will take the inverse of $i_{\gamma, \gamma \gamma^{\prime}}$ and $e v_{\gamma, \gamma \gamma^{\prime}, \gamma \gamma^{\prime} \gamma^{\prime \prime}}$ to construct the corresponding data for the stack of Hopf algebras.

${ }^{3}$ Deformation quantization here means that $\mathbb{A}_{\gamma} / \hbar \mathbb{A}_{\gamma}=\mathbb{O}_{G_{\gamma}^{*}}$, and $\frac{1}{\hbar}[\cdot, \cdot]_{*_{\gamma}}=\{\cdot, \cdot\}_{\gamma}+O(\hbar)$.
} 
is convergent, where $\sum_{i>1} B_{i}(x, y)$ is the Baker-Campbell-Hausdorff $(\mathrm{BCH})$ series specialized to the Poisson bracket of $\mathfrak{m}_{\left(G^{*}\right)^{k}}^{2}$. The $\star$ product defines a group structure on $\mathfrak{m}_{\left(G^{*}\right)^{k}}^{2}$.

A useful technical lemma was proved in [Enriquez et al. 2003, page 2477] for $m_{\mathfrak{g}^{*}}$ and is still true for $m_{G^{*}}$ :

Lemma 3.1. For any $k \geq 1$ and $n \geq 2, f, h \in \mathfrak{m}_{\left(G^{*}\right)^{k}}^{2}$ and $g \in \mathfrak{m}_{\left(G^{*}\right)^{k}}^{n}$, one has

$$
f \star(h+g)=f \star h+g \text { and }(f+g) \star h=f \star h+g \text { modulo } \mathfrak{m}_{\left(G^{*}\right)^{k}}^{n+1}
$$

When $\left(\mathfrak{g}, \theta_{\mathfrak{g}}, \delta_{\mathfrak{g}}, f\right)$ is a $\Gamma$ Lie bialgebra we thus get a collection of Lie bialgebras and so a collection $\left(\mathscr{O}_{G_{\gamma}^{*}}, m_{\gamma}, \Delta_{\gamma},\{\cdot, \cdot\}_{\gamma}\right)_{\gamma \in \Gamma}$ of Poisson bialgebras. We will denote by $\star_{\gamma}$ the corresponding $\mathrm{BCH}$ products.

"Lifts" and functional equations. We will now construct "lifts" $\tilde{f}_{\gamma, \gamma \gamma^{\prime}} \in \mathfrak{m}_{G^{*}}^{\widehat{\otimes}}$ of the elements $\Lambda^{2}\left(\theta_{\gamma}\right)\left(f_{\gamma^{\prime}}\right), \gamma, \gamma^{\prime} \in \Gamma$ that will satisfy similar relation as $\mathrm{F}_{\gamma, \gamma \gamma^{\prime}}$ in Proposition 1.5. The proof in this subsection is a direct generalization of the results in [Enriquez and Halbout 2007], and some parts are transcribed almost verbatim.

If $f \in \mathcal{O}_{G^{*}}^{\widehat{\otimes} n}$ and $P_{1}, \ldots, P_{m}$ are disjoint subsets of $\{1, \ldots, m\}$, one defines $f^{P_{1}, \ldots, P_{n}}$ using the coproduct of $\mathrm{O}_{G^{*}}$ :

Definition 3.2. If $I_{1}, \ldots, I_{m}$ are disjoint ordered subsets of $\{1, \ldots, n\},(U, \Delta)$ is a Hopf algebra, and $a \in U^{\otimes m}$, we define

$$
a^{I_{1}, \ldots, I_{n}}=\sigma_{I_{1}, \ldots, I_{m}} \circ\left(\Delta^{\left|I_{1}\right|} \otimes \cdots \otimes \Delta^{\left|I_{n}\right|}\right)(a),
$$

with $\Delta^{(1)}=\mathrm{id}, \quad \Delta^{(2)}=\Delta$, and $\Delta^{(n+1)}=\left(\mathrm{id}^{\otimes n-1} \otimes \Delta\right) \circ \Delta^{(n)}$. Here

$$
\sigma_{I_{1}, \ldots, I_{m}}: U^{\otimes \sum_{i}\left|I_{i}\right|} \rightarrow U^{\otimes n}
$$

is the morphism corresponding to the map $\left\{1, \ldots, \sum_{i}\left|I_{i}\right|\right\} \rightarrow\{1, \ldots, n\}$ taking $\left(1, \ldots,\left|I_{1}\right|\right)$ to $I_{1},\left(\left|I_{1}\right|+1, \ldots,\left|I_{1}\right|+\left|I_{2}\right|\right)$ to $I_{2}$, and so on.

When $U$ is cocommutative, this definition depends only on the underlying sets $I_{1}, \ldots, I_{m}$.

Proposition 3.3. Let $\gamma, \gamma^{\prime}$ be in $\Gamma$. Then there exists $\tilde{f}_{\gamma, \gamma \gamma^{\prime}}$ in $m_{G^{*}}^{\widehat{\otimes} 2}$, the image of which in $\mathfrak{g}^{\otimes 2}$ under the square of the projection $\mathfrak{m}_{G^{*}} \rightarrow \mathfrak{m}_{G^{*}} / \mathfrak{m}_{G^{*}}^{2}=\mathfrak{g}$ equals $\bigwedge^{2}\left(\theta_{\gamma}\right)\left(f_{\gamma^{\prime}}\right)$, and such that

$$
\left(\tilde{f}_{\gamma, \gamma \gamma^{\prime}} \otimes 1\right) \star_{\gamma}\left(\Delta_{\gamma} \otimes \mathrm{id}\right)\left(\tilde{f}_{\gamma, \gamma \gamma^{\prime}}\right)=\left(1 \otimes \tilde{f}_{\gamma, \gamma \gamma^{\prime}}\right) \star_{\gamma}\left(\mathrm{id} \otimes \Delta_{\gamma}\right)\left(\tilde{f}_{\gamma, \gamma \gamma^{\prime}}\right) .
$$

The element $\tilde{f}_{\gamma, \gamma \gamma^{\prime}}$ is unique up to the action of $\mathfrak{m}_{G^{*}}^{2}$ by $\lambda \cdot \tilde{f}=\lambda^{1} \star_{\gamma} \lambda^{2} \star_{\gamma} \tilde{f} \star_{\gamma}(-\lambda)^{12}$. We will call $\tilde{f}$ a twist for $\Delta_{\gamma}$. 
Proof. Let us construct $\tilde{f}_{\gamma, \gamma \gamma^{\prime}}$ by induction, by constructing a convergent sequence $\tilde{f}^{N} \in \mathfrak{m}_{G^{*}}^{\widehat{\otimes} 2}(N \geq 2)$ satisfying (1) in $\mathfrak{m}_{G^{*}}^{\widehat{\otimes} 3} /\left(\mathfrak{m}_{G^{*}}^{\widehat{\otimes} 3} \cap \mathfrak{m}_{\left(G^{*}\right)^{3}}^{N}\right)$, where $\mathfrak{m}_{\left(G^{*}\right)^{3}}^{N}$ is the $N$-th power of $\mathfrak{m}_{\left(G^{*}\right)^{3}}$. When $N=3$, we take $\tilde{f}^{2}$ to be any lift of $\Lambda^{2}\left(\theta_{\gamma}\right)\left(f_{\gamma^{\prime}}\right)$ to $\mathfrak{m}_{G^{*}}^{\widehat{\otimes} 2}$; then (1) is automatically satisfied.

Let $N$ be an integer no less than 3; assume that we have constructed $\tilde{f}^{N}$ in $\mathfrak{m}_{G^{*}}^{\widehat{\otimes}}$ satisfying (1) in $\mathfrak{m}_{G^{*}}^{\widehat{\otimes} 3} /\left(\mathfrak{m}_{G^{*}}^{\widehat{\otimes}} \cap \mathfrak{m}_{\left(G^{*}\right)^{3}}^{N}\right)$. Set $\alpha_{1,2,3}^{N}:=\tilde{f}_{1,2}^{N} \star_{\gamma} \tilde{f}_{12,3}^{N}-\tilde{f}_{2,3}^{N} \star_{\gamma} \tilde{f}_{1,23}^{N}$. Then $\alpha_{1,2,3}^{N}$ belongs to $\mathfrak{m}_{G^{*}}^{\widehat{\otimes} 3} \cap \mathfrak{m}_{\left(G^{*}\right)^{3}}^{N}$, and in $\mathfrak{m}_{G^{*}}^{\widehat{\otimes} 4} /\left(\mathfrak{m}_{G^{*}}^{\widehat{\otimes} 4} \cap \mathfrak{m}_{\left(G^{*}\right)^{4}}^{N+1}\right)$, we have the equalities

$$
\begin{aligned}
& \alpha_{12,3,4}^{N}=\tilde{f}_{1,2}^{N} \star_{\gamma} \alpha_{12,3,4}^{N}=\tilde{f}_{1,2}^{N} \star_{\gamma} \tilde{f}_{12,3}^{N} \star_{\gamma} \tilde{f}_{123,4}^{N}-\tilde{f}_{1,2}^{N} \star_{\gamma} \tilde{f}_{3,4}^{N} \star_{\gamma} \tilde{f}_{12,34}^{N} \\
& =\alpha_{1,2,3}^{N}+\tilde{f}_{2,3}^{N} \star_{\gamma} \tilde{f}_{1,23}^{N} \star_{\gamma} \tilde{f}_{123,4}^{N}-\tilde{f}_{3,4}^{N} \star_{\gamma} \tilde{f}_{1,2}^{N} \star_{\gamma} \tilde{f}_{12,34}^{N} \\
& \text { (using Lemma 3.1) } \\
& =\alpha_{1,2,3}^{N}+\tilde{f}_{2,3}^{N} \star_{\gamma} \tilde{f}_{1,23}^{N} \star \tilde{f}_{123,4}^{N}-\tilde{f}_{3,4}^{N} \star\left(\tilde{f}_{2,34}^{N} \star_{\gamma} \tilde{f}_{1,234}^{N}+\alpha_{1,2,34}^{N}\right) \\
& \text { (using Lemma } 3.1 \text { and the definition of } \alpha_{1,2,34}^{N} \text { ) } \\
& =\alpha_{1,2,3}^{N}+\tilde{f}_{2,3}^{N} \star_{\gamma}\left(\alpha_{1,23,4}^{N}+\tilde{f}_{23,4}^{N} \star_{\gamma} \tilde{f}_{1,234}^{N}\right) \\
& -\alpha_{1,2,34}^{N}-\tilde{f}_{3,4}^{N} \star_{\gamma} \tilde{f}_{2,34}^{N} \star_{\gamma} \tilde{f}_{1,234}^{N} \\
& \text { (using the definition of } \alpha_{1,23,4}^{N} \text { and Lemma 3.1) } \\
& =\alpha_{1,2,3}^{N}+\alpha_{1,23,4}^{N}+\left(\tilde{f}_{3,4}^{N} \star_{\gamma} \tilde{f}_{2,34}^{N}+\alpha_{2,3,4}^{N}\right) \star_{\gamma} \tilde{f}_{1,234}^{N} \\
& -\alpha_{1,2,34}^{N}-\tilde{f}_{3,4}^{N} \star_{\gamma} \tilde{f}_{2,34}^{N} \star_{\gamma} \tilde{f}_{1,234}^{N} \\
& \text { (using the definition of } \alpha_{2,3,4}^{N} \text { and Lemma 3.1) } \\
& =\alpha_{1,2,3}^{N}+\alpha_{1,23,4}^{N}-\alpha_{1,2,34}^{N}+\alpha_{2,3,4}^{N}
\end{aligned}
$$

Let us denote by $\bar{\alpha}^{N}$ the image of $\alpha^{N}$ in $\left(\mathfrak{m}_{\mathfrak{g}^{*}}^{\widehat{\otimes} 3} \cap \mathfrak{m}_{\left(\mathfrak{g}^{*}\right)^{3}}^{N}\right) /\left(\mathfrak{m}_{\mathfrak{g}^{*}}^{\widehat{\otimes} 3} \cap \mathfrak{m}_{\left(\mathfrak{g}^{*}\right)^{3}}^{N+1}\right)=$ $\left(S^{>0}(\mathfrak{g})^{\otimes 3}\right)_{N}$. Then we get

$$
\bar{\alpha}_{12,3,4}^{N}+\bar{\alpha}_{1,2,34}^{N}=\bar{\alpha}_{1,2,3}^{N}+\bar{\alpha}_{1,23,4}^{N}+\bar{\alpha}_{2,3,4}^{N},
$$

meaning that $\bar{\alpha}$ is a cocycle for the subcomplex $\left(S^{>0}(\mathfrak{g})^{\otimes \cdot}, d\right)$ of the co-Hochschild complex. By using [Drinfeld 1989, Proposition 3.11], one proves that the $k$-th cohomology group of this subcomplex is $\Lambda^{k}(\mathfrak{g})$, and that the antisymmetrization map coincides with the canonical projection from the space of cocycles to the cohomology group. For $N=3$, the equations of Definition $1.2 \operatorname{imply} \operatorname{Alt}\left(\bar{\alpha}^{3}\right)=0$, and hence $\bar{\alpha}^{3}$ is the coboundary of an element $\bar{\beta}^{3} \in\left(S^{>0}(\mathfrak{g})^{\otimes 2}\right)^{3}$, and $\bar{\alpha}^{N}$ for $N>3$ is the coboundary of an element $\bar{\beta}^{N} \in\left(S^{>0}(\mathfrak{g})^{\otimes 2}\right)^{N}$ since the degree $N$ part of the cohomology vanishes. We then set $\tilde{f}^{N+1}:=\tilde{f}^{N}+\beta^{N}$, where $\beta^{N} \in \mathfrak{m}_{G^{*}}^{\widehat{\otimes}^{2}} \cap \mathfrak{m}_{\left(G^{*}\right)^{2}}^{N}$ is a representative of $\bar{\beta}^{N}$. Then this $\tilde{f}^{N+1}$ satisfies (1) in $\mathfrak{m}_{G *}^{\widehat{\otimes} 3} /\left(\mathfrak{m}_{G^{*}}^{\widehat{\otimes} 3} \cap \mathfrak{m}_{\left(G^{*}\right)^{3}}^{N+1}\right.$.

The sequence $\left(\tilde{f}^{N}\right)^{N \geq 2}$ has a limit $\tilde{f}$, which then satisfies (1).

The second part of the theorem can be proved in the same way or by analyzing the choices for $\bar{\beta}^{N}$ in the proof above. 


\section{Isomorphism of formal Poisson groups $G_{\gamma}^{*} \simeq G_{\gamma \gamma^{\prime}}^{*}$.}

Proposition 3.4. Let $\gamma, \gamma^{\prime} \in \Gamma$ and let $G_{\gamma}^{*}$ and $G_{\gamma \gamma^{\prime}}^{*}$ be the formal Poisson-Lie groups associated to the corresponding Lie cobrackets. There exists an isomorphism of Poisson algebras $j_{\gamma, \gamma \gamma^{\prime}}: \mathbb{O}_{G_{\gamma}^{*}} \simeq \mathbb{O}_{G_{\gamma \gamma^{\prime}}^{*}}$.

Proof. Let $P: \bigwedge^{2}\left(\mathscr{O}_{G_{\gamma}^{*}}\right) \rightarrow \mathcal{O}_{G_{\gamma}^{*}}$ be the Poisson bracket on $\mathscr{O}_{G_{\gamma}^{*}}$ corresponding to the Lie-Poisson Poisson structure on $G_{\gamma}^{*}$. Then $\left(\sigma_{G_{\gamma}^{*}}, m_{0}, P, \Delta_{\gamma}\right)$ is a Poisson formal series Hopf (PFSH) algebra; it corresponds to the formal Poisson-Lie group $G_{\gamma}^{*}$ equipped with its Lie-Poisson structure.

Set $\tilde{f}_{\gamma, \gamma \gamma^{\prime}}^{\star} \Delta_{\gamma}(a)=\tilde{f}_{\gamma, \gamma \gamma^{\prime} \star_{\gamma}} \Delta_{\gamma}(a) \star_{\gamma}\left(-\tilde{f}_{\gamma, \gamma \gamma^{\prime}}\right)$ for any $a \in \tilde{O}_{G_{\gamma}^{*}}$. It follows from the fact that $\tilde{f}_{\gamma, \gamma \gamma^{\prime}}$ satisfies Equation (1) that $\left(\mathscr{O}_{G_{\gamma}^{*}}, m_{0}, P, \tilde{f}_{\gamma, \gamma \gamma^{\prime}}^{\star} \Delta_{\gamma}\right)$ is a PFSH algebra.

Let us denote by PFSHA and LBA the categories of PSFH algebras and Lie bialgebras. We have a category equivalence $c:$ PFSHA $\rightarrow$ LBA, taking $(\mathcal{O}, m, P, \Delta)$ to the Lie bialgebra $(\mathfrak{c}, \mu, \delta)$, where $\mathfrak{c}:=\mathfrak{m} / \mathfrak{m}^{2}$ (here $\mathfrak{m} \subset \mathcal{O}$ is the maximal ideal), the Lie cobracket of $\mathfrak{c}$ is induced by $\Delta-\Delta^{2,1}: \mathfrak{m} \rightarrow \Lambda^{2}(\mathfrak{m})$, and the Lie bracket of $\mathfrak{c}$ is induced by the Poisson bracket $P: \Lambda^{2}(\mathfrak{m}) \rightarrow \mathfrak{m}$. The inverse of the functor $c$ takes $(\mathfrak{c}, \mu, \delta)$ to $O=\hat{S}(\mathfrak{c})$ equipped with its usual product; $\Delta$ depends only on $\delta$ and $P$ depends on $(\mu, \delta)$.

Then $c$ restricts to a category equivalence $c_{\mathrm{fd}}: \mathbf{P F S H A} \mathbf{A}_{\mathrm{fd}} \rightarrow \mathbf{L B A} \mathbf{A}_{\mathrm{fd}}$ of subcategories of finite-dimensional objects (in the case of PFSH, we say that 0 is finite-dimensional if and only if $\mathfrak{m} / \mathfrak{m}^{2}$ is).

Let dual : $\mathbf{L} \mathbf{B} \mathbf{A}_{\mathrm{fd}} \rightarrow \mathbf{L B A} \mathbf{A}_{\mathrm{fd}}$ be the duality functor. It is a category antiequivalence; we have dual $(\mathfrak{g}, \mu, \delta)=\left(\mathfrak{g}^{*}, \delta^{t}, \mu^{t}\right)$. Then dual $\circ c_{\mathrm{fd}}: \mathbf{P F S H A}_{\mathrm{fd}} \rightarrow \mathbf{L B A}_{\mathrm{fd}}$ is a category antiequivalence. Its inverse is the usual functor $\mathfrak{g} \mapsto U(\mathfrak{g})^{*}$. If $G$ is the formal Poisson-Lie group with Lie bialgebra $\mathfrak{g}$, one sets $\mathscr{O}_{G}=U(\mathfrak{g})^{*}$.

Let us apply the functor $c$ to $\left(\mathscr{O}_{G_{\gamma}^{*}}, m_{0}, P, \tilde{f}_{\gamma, \gamma \gamma^{\prime}}^{\star} \Delta_{\gamma}\right)$. We obtain $\mathfrak{c}=\mathfrak{m} / \mathfrak{m}^{2}=\mathfrak{g}$; the Lie bracket is unchanged with respect to the case $\tilde{f}_{\gamma, \gamma \gamma^{\prime}}=0$, so it is the Lie bracket of $\mathfrak{g}$; the Lie cobracket is $\delta_{\gamma \gamma^{\prime}}(x)=\delta_{\gamma}+\left[\bigwedge^{2}\left(\theta_{\gamma}\right)\left(f_{\gamma^{\prime}}\right), x \otimes 1+1 \otimes x\right]$ since the reduction of $\tilde{f}_{\gamma, \gamma \gamma^{\prime}}$ modulo $\left(\mathfrak{m}_{G_{\gamma}^{*}}\right)^{2} \widehat{\otimes} \mathfrak{m}_{G_{\gamma}^{*}}+\mathfrak{m}_{G_{\gamma}^{*}} \widehat{\otimes}\left(\mathfrak{m}_{G_{\gamma}^{*}}\right)^{2}$ is equal to $\bigwedge^{2}\left(\theta_{\gamma}\right)\left(f_{\gamma^{\prime}}\right)$.

Then applying dual $\circ c_{\mathrm{fd}}$ to $\left(\mathscr{O}_{G_{\gamma}^{*}}, m_{0}, P, \tilde{f}_{\gamma, \gamma \gamma^{\prime}}^{\star} \Delta_{\gamma}\right)$, we obtain the Lie bialgebra $\left(\mathfrak{g}^{*}, \delta_{\gamma \gamma^{\prime}}\right)$. So this PFSH algebra is isomorphic to the PFSH algebra of the formal Poisson-Lie group $G_{\gamma \gamma^{\prime}}^{*}$. Let us call such a PFSH algebra morphism $j_{\gamma, \gamma \gamma^{\prime}}$.

In particular, the Poisson algebras $\mathscr{O}_{G_{\gamma}^{*}}$ and $\mathscr{O}_{G_{\gamma \gamma^{\prime}}^{*}}$ are isomorphic.

Remark 3.5. It is easy to check that the map $\mathfrak{g}=\mathfrak{m}_{G_{\gamma}^{*}} / \mathfrak{m}_{G_{\gamma}^{*}}^{2} \rightarrow \mathfrak{m}_{G_{\gamma \gamma^{\prime}}^{*}} / \mathfrak{m}_{G_{\gamma \gamma^{\prime}}^{*}}^{2}=\mathfrak{g}$ induced by the isomorphism $j_{\gamma, \gamma \gamma^{\prime}}$ is the identity.

Remark 3.6. We have proved a result stronger than the existence of a Poisson algebra morphism $j_{\gamma, \gamma \gamma^{\prime}}: \mathbb{O}_{G_{\gamma}^{*}} \simeq \mathscr{O}_{G_{\gamma \gamma^{\prime}}^{*}}$. This morphism intertwines the coproducts as

$$
\Delta_{\gamma \gamma^{\prime}}=j_{\gamma, \gamma \gamma^{\prime}}^{\otimes 2} \circ \tilde{f}_{\gamma, \gamma \gamma^{\prime}}^{\star} \Delta_{\gamma} \circ j_{\gamma, \gamma \gamma^{\prime}}^{-1}
$$




\section{Composition of equivalences.}

Lemma 3.7. For $\gamma, \gamma^{\prime}$ in $\Gamma$, the element $\left(j_{\gamma, \gamma \gamma^{\prime}}^{\otimes 2}\right)^{-1}\left(\tilde{f}_{\gamma \gamma^{\prime}, \gamma \gamma^{\prime} \gamma^{\prime \prime}}\right) \star_{\gamma} \tilde{f}_{\gamma, \gamma \gamma^{\prime}}$ is a solution of the equation

$$
(\tilde{g} \otimes 1) \star_{\gamma}\left(\Delta_{\gamma} \otimes \mathrm{id}\right)(\tilde{g})=(1 \otimes \tilde{g}) \star_{\gamma}\left(\mathrm{id} \otimes \Delta_{\gamma}\right)(\tilde{g}) .
$$

Proof. One can check this directly. Notice that $\tilde{f}_{\gamma \gamma^{\prime}, \gamma \gamma^{\prime} \gamma^{\prime \prime}}$ is a twist for $\Delta_{\gamma \gamma^{\prime}}$. Therefore $\left(j_{\gamma, \gamma \gamma^{\prime}}^{\otimes 2}\right)^{-1}\left(\tilde{f}_{\gamma \gamma^{\prime}, \gamma \gamma^{\prime} \gamma^{\prime \prime}}\right)$ is a twist for

$$
\left(j_{\gamma, \gamma \gamma^{\prime}}^{\otimes 2}\right)^{-1} \circ \Delta_{\gamma \gamma^{\prime}} \circ j_{\gamma, \gamma \gamma^{\prime}}=\tilde{f}_{\gamma, \gamma \gamma^{\prime}}^{\star} \Delta_{\gamma} .
$$

Accordingly the element $\left(j_{\gamma, \gamma \gamma^{\prime}}^{\otimes 2}\right)^{-1}\left(\tilde{f}_{\gamma \gamma^{\prime}, \gamma \gamma^{\prime} \gamma^{\prime \prime}}\right) \star_{\gamma} \tilde{f}_{\gamma, \gamma \gamma^{\prime}}$ is a twist for $\Delta_{\gamma}$.

Note that the image of $\left(j_{\gamma, \gamma \gamma^{\prime}}^{\otimes 2}\right)^{-1}\left(\tilde{f}_{\gamma \gamma^{\prime}, \gamma \gamma^{\prime} \gamma^{\prime \prime}}\right) \star_{\gamma} \tilde{f}_{\gamma, \gamma \gamma^{\prime}}$ under the square of the projection $\mathfrak{m}_{G^{*}} \rightarrow \mathfrak{m}_{G^{*}} / \mathfrak{m}_{G^{*}}^{2}=\mathfrak{g}$ equals

$$
\bigwedge^{2}\left(\theta_{\gamma}\right)\left(f_{\gamma^{\prime}}\right)+\Lambda^{2}\left(\theta_{\gamma \gamma^{\prime}}\right)\left(f_{\gamma^{\prime \prime}}\right)=\Lambda^{2}\left(\theta_{\gamma}\right)\left(f_{\gamma^{\prime}}+\Lambda^{2}\left(\theta_{\gamma^{\prime}}\right)\left(f_{\gamma^{\prime \prime}}\right)\right)=\Lambda^{2}\left(\theta_{\gamma}\right)\left(f_{\gamma^{\prime} \gamma^{\prime \prime}}\right) .
$$

Thanks to Proposition 3.3, there exists an element $u_{\gamma, \gamma \gamma^{\prime}, \gamma \gamma^{\prime} \gamma^{\prime \prime}}$ in $1+\mathfrak{m}_{G^{*}}^{2}$ such that

$$
\tilde{f}_{\gamma, \gamma \gamma^{\prime} \gamma^{\prime \prime}}=u_{\gamma, \gamma \gamma^{\prime}, \gamma \gamma^{\prime} \gamma^{\prime \prime}}^{\otimes 2} \star_{\gamma}\left(j_{\gamma, \gamma \gamma^{\prime}}^{\otimes 2}\right)^{-1}\left(\tilde{f}_{\gamma \gamma^{\prime}, \gamma \gamma^{\prime} \gamma^{\prime \prime}}\right) \star_{\gamma} \tilde{f}_{\gamma, \gamma \gamma^{\prime} \star_{\gamma}} \Delta_{\gamma}\left(u_{\gamma, \gamma \gamma^{\prime}, \gamma \gamma^{\prime} \gamma^{\prime \prime}}\right)^{-1} .
$$

Finally, in the previous section, we defined $j_{\gamma, \gamma \gamma^{\prime}}, j_{\gamma \gamma^{\prime}, \gamma \gamma^{\prime} \gamma^{\prime \prime}}$ and $j_{\gamma, \gamma \gamma^{\prime} \gamma^{\prime \prime}}$ such that

$$
\begin{aligned}
& \Delta_{\gamma \gamma^{\prime} \gamma^{\prime \prime}}=j_{\gamma, \gamma \gamma^{\prime} \gamma^{\prime \prime}}^{\otimes 2} \circ \tilde{f}_{\gamma, \gamma \gamma^{\prime} \gamma^{\prime \prime}}^{\star} \Delta_{\gamma} \circ j_{\gamma, \gamma \gamma^{\prime} \gamma^{\prime \prime}}^{-1} \\
& =j_{\gamma, \gamma \gamma^{\prime} \gamma^{\prime \prime}}^{\otimes 2} \\
& \circ\left(u_{\gamma, \gamma \gamma^{\prime}, \gamma \gamma^{\prime} \gamma^{\prime \prime}}^{\otimes \star_{\gamma}}\left(j_{\gamma, \gamma \gamma^{\prime}}^{\otimes 2}\right)^{-1}\left(\tilde{f}_{\gamma \gamma^{\prime}, \gamma \gamma^{\prime} \gamma^{\prime \prime}}\right) \star_{\gamma} \tilde{f}_{\gamma, \gamma \gamma^{\prime}} \star_{\gamma} \Delta_{\gamma}\left(u_{\gamma, \gamma \gamma^{\prime}, \gamma \gamma^{\prime} \gamma^{\prime \prime}}\right)^{-1}\right)^{\star} \Delta_{\gamma} \\
& \circ j_{\gamma, \gamma \gamma^{\prime} \gamma^{\prime \prime}}^{-1}
\end{aligned}
$$

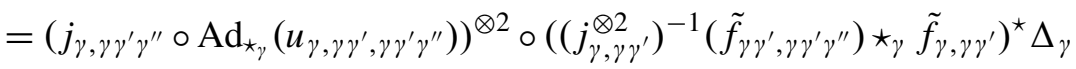

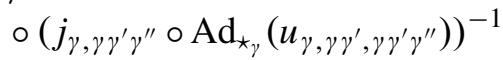

$$
\begin{aligned}
& =\left(j_{\gamma, \gamma \gamma^{\prime} \gamma^{\prime \prime}} \circ \operatorname{Ad}_{\star_{\gamma}}\left(u_{\gamma, \gamma \gamma^{\prime}, \gamma \gamma^{\prime} \gamma^{\prime \prime}}\right) \circ j_{\gamma, \gamma \gamma^{\prime}}^{-1} \circ j_{\gamma \gamma^{\prime}, \gamma \gamma^{\prime} \gamma^{\prime \prime}}^{-1}\right)^{\otimes 2} \circ \Delta_{\gamma \gamma^{\prime} \gamma^{\prime \prime}} \\
& \circ\left(j_{\gamma, \gamma \gamma^{\prime} \gamma^{\prime \prime}} \circ \operatorname{Ad}_{\star_{\gamma}}\left(u_{\gamma, \gamma \gamma^{\prime}, \gamma \gamma^{\prime} \gamma^{\prime \prime}}\right) \circ j_{\gamma, \gamma \gamma^{\prime}}^{-1} \circ j_{\gamma \gamma^{\prime}, \gamma \gamma^{\prime} \gamma^{\prime \prime}}^{-1}\right)^{-1} \text {. }
\end{aligned}
$$

By the equivalence $c_{\mathrm{fd}}$ between the category $\mathbf{P F S H A} \mathbf{A}_{\mathrm{fd}}$ and $\mathbf{L B A} \mathbf{A}_{\mathrm{fd}}$, we get

$$
j_{\gamma, \gamma \gamma^{\prime} \gamma^{\prime \prime}}=j_{\gamma \gamma^{\prime}, \gamma \gamma^{\prime} \gamma^{\prime \prime}} \circ j_{\gamma, \gamma \gamma^{\prime}} \circ \operatorname{Ad}_{\star \gamma}\left(u_{\gamma, \gamma \gamma^{\prime}, \gamma \gamma^{\prime} \gamma^{\prime \prime}}^{-1}\right) \text {. }
$$

\section{Cocycle relation for the $u_{\gamma, \gamma \gamma^{\prime}, \gamma \gamma^{\prime} \gamma^{\prime \prime}}$.}

Proposition 3.8. For any $\gamma, \gamma^{\prime}, \gamma^{\prime \prime}, \gamma^{\prime \prime \prime}$ in $\Gamma$, we have

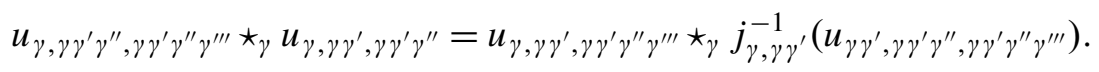


Proof. To shorten the notation, we will write $\tilde{f}_{1,2}$ for $\tilde{f}_{\gamma, \gamma \gamma^{\prime}}, \tilde{f}_{2,3}$ for $\tilde{f}_{\gamma \gamma^{\prime}, \gamma \gamma^{\prime} \gamma^{\prime \prime}}$ and so on, and the same for the $j_{.,}$. and the $u_{,, .,}$. We will omit the BCH product $\star_{\gamma}$ and write $\star$ for the product $\star_{\gamma \gamma^{\prime}}, \Delta_{0}$ for the coproduct $\Delta_{\gamma}$, and $\Delta$ for the coproduct $\Delta_{\gamma \gamma^{\prime}}$. We will also write $j(\cdot)$ instead of $j^{\otimes 2}(\cdot)$ when no confusion is possible.

We have by definition $\tilde{f}_{1,4} \Delta_{0} u_{1,3,4}=u_{1,3,4}^{\otimes 2} j_{1,3}^{-1}\left(\tilde{f}_{3,4}\right) \tilde{f}_{1,3}$. Multiplying this on the right by $\Delta_{0} u_{1,2,3}$ and using the fact that $\tilde{f}_{1,3} \Delta_{0} u_{1,2,3}=u_{1,2,3}^{\otimes 2} j_{1,2}^{-1}\left(\tilde{f}_{2,3}\right) \tilde{f}_{1,2}$, we get

$$
\tilde{f}_{1,4} \Delta_{0} u_{1,3,4} \Delta_{0} u_{1,2,3}=u_{1,3,4}^{\otimes 2} j_{1,3}^{-1}\left(\tilde{f}_{3,4}\right) u_{1,2,3}^{\otimes 2} j_{1,2}^{-1}\left(\tilde{f}_{2,3}\right) \tilde{f}_{1,2} .
$$

Using now that $j_{1,3}^{-1}(\cdot) u_{1,2,3}=u_{1,2,3} j_{1,2}^{-1} \circ j_{2,3}^{-1}(\cdot)$, we get

$$
\tilde{f}_{1,4} \Delta_{0} u=u^{\otimes 2} j_{1,2}^{-1} \circ j_{2,3}^{-1}\left(\tilde{f}_{3,4}\right) j_{1,2}^{-1}\left(\tilde{f}_{2,3}\right) \tilde{f}_{1,2},
$$

where $u=u_{1,3,4} u_{1,2,3}$. On the other hand, we have

$$
\tilde{f}_{2,4} \star \Delta u_{2,3,4}=u_{2,3,4}^{\otimes 2} \star j_{2,3}^{-1}\left(\tilde{f}_{3,4}\right) \star \tilde{f}_{2,3} .
$$

Using the Poisson algebra morphism $j_{1,2}$ and that $j_{1,2}^{-1} \circ \Delta=\tilde{f}_{1,2} \Delta_{0}\left(j_{1,2}^{-1}(\cdot)\right) \tilde{f}_{1,2}^{-1}$, we get

$$
j_{1,2}^{-1}\left(\tilde{f}_{2,4}\right) \tilde{f}_{1,2} \Delta_{0}\left(j_{1,2}^{-1}\left(u_{2,3,4}\right)\right) \tilde{f}_{1,2}^{-1}=j_{1,2}^{-1}\left(u_{2,3,4}^{\otimes 2}\right) j_{1,2}^{-1} \circ j_{2,3}^{-1}\left(\tilde{f}_{3,4}\right) j_{1,2}^{-1}\left(\tilde{f}_{2,3}\right) .
$$

From $\tilde{f}_{1,4} \Delta_{0} u_{1,2,4}=u_{1,2,4}^{\otimes 2} j_{1,2}^{-1}\left(\tilde{f}_{2,4}\right) \tilde{f}_{1,2}$, using (4) we get

$$
\tilde{f}_{1,4} \Delta_{0}\left(u^{\prime}\right)=\left(u^{\prime}\right)^{\otimes 2} j_{1,2}^{-1} \circ j_{2,3}^{-1}\left(\tilde{f}_{3,4}\right) j_{1,2}^{-1}\left(\tilde{f}_{2,3}\right) \tilde{f}_{1,2},
$$

where $u^{\prime}=u_{1,2,4} j_{1,2}^{-1}\left(u_{2,3,4}\right)$. Then (3) and (5) imply that if $w=u\left(u^{\prime}\right)^{-1}$, then $\tilde{f}_{1,4} \Delta_{0}(w)=w \tilde{f}_{1,4}$, and so if $w^{\prime}=j_{1,4}(w)$, then $\Delta_{0}\left(w^{\prime}\right)=w^{\prime}$. Recall that $w^{\prime} \in 1+m_{G^{*}}^{2}$ by similar properties of $u_{i, j, k}$. Suppose that $w^{\prime} \neq 1$ and set $i \geq 2$ the largest possible $i$ such that $w^{\prime}$ is in $1+m_{G^{*}}^{i}$ but not in $1+m_{G^{*}}^{i+1}$. Let $\bar{w}^{\prime}$ be the projection of $w^{\prime}$ in $m_{G^{*}}^{i} / m_{G^{*}}^{i+1}$. The relation $\Delta_{0}\left(w^{\prime}\right)=w^{\prime}$ implies that $\bar{w}^{\prime}$ is in $\mathfrak{g}$ and so in $m_{G^{*}}^{1}$ which is a contradiction. Thus we have proved that $w=w^{\prime}=1$ and so that $u=u^{\prime}$.

\section{Quantization}

Duality of QUE and QFSH algebras. In this subsection, we recall some facts from [Drinfeld 1987], whose proofs can be found in [Gavarini 2002]. Let us denote by QUE the category of quantized universal enveloping (QUE) algebras and by QFSH the category of quantized formal series Hopf (QFSH) algebras. We denote by $\mathbf{Q U E} \mathbf{E}_{\mathrm{fd}}$ and $\mathbf{Q F S H} \mathbf{f d}_{\mathrm{fd}}$ the subcategories corresponding to finite dimensional Lie bialgebras.

We have contravariant functors

$$
\mathbf{Q U E}_{\mathrm{fd}} \rightarrow \mathbf{Q F S H}_{\mathrm{fd}}, \quad U \mapsto U^{*} \quad \text { and } \quad \mathbf{Q F S H}_{\mathrm{fd}} \rightarrow \mathbf{Q U E}_{\mathrm{fd}}, \quad 0 \mapsto 0^{\circ}
$$


These functors are inverse to each other. Here $U^{*}$ is the full topological dual of $U$, that is, the space of all continuous (for the $\hbar$-adic topology) $\mathbb{\llbracket} \llbracket \rrbracket$-linear maps $U \rightarrow \mathbb{K} \llbracket \hbar \rrbracket$, and $0^{\circ}$ is the space of continuous $\mathbb{K} \llbracket \hbar \rrbracket$-linear forms $O \rightarrow \mathbb{K} \llbracket \hbar \rrbracket$, where $\mathbb{O}$ is equipped with the $\mathfrak{m}$-adic topology (here $\mathfrak{m} \subset \mathbb{O}$ is the maximal ideal).

We also have covariant functors

$$
\mathbf{Q U E} \rightarrow \text { QFSH, } \quad U \mapsto U^{\prime} \text { and } \mathbf{Q F S H} \rightarrow \mathbf{Q U E}, \quad 0 \mapsto 0^{\vee} .
$$

These functors are also inverse to each other. Here $U^{\prime}$ is a subalgebra of $U$, while $\mathrm{O}^{\vee}$ is the $\hbar$-adic completion of $\sum_{k \geq 0} \hbar^{-k} \mathfrak{m}^{k} \subset \mathcal{O}[1 / \hbar]$.

We also have canonical isomorphisms $\left(U^{\prime}\right)^{\circ} \simeq\left(U^{*}\right)^{\vee}$ and $\left(O^{\vee}\right)^{*} \simeq\left(O^{\circ}\right)^{\prime}$.

If $\mathfrak{a}$ is a finite-dimensional Lie bialgebra and $U=U_{\hbar}(\mathfrak{a})$ is a QUE algebra quantizing $\mathfrak{a}$, then $U^{*}=\mathbb{O}_{A, \hbar}$ is a QFSH algebra quantizing the Poisson-Lie group $A$, with Lie bialgebra $\mathfrak{a}$, and $U^{\prime}=\mathbb{O}_{A^{*}, \hbar}$ is a QFSH algebra quantizing the Poisson-Lie group $A^{*}$, with Lie bialgebra $\mathfrak{a}^{*}$. If now $\mathrm{O}=\mathrm{O}_{A, \hbar}$ is a QFSH algebra quantizing $A$, then $0^{\circ}=U_{\hbar}(\mathfrak{a})$ is a QUE algebra quantizing $\mathfrak{a}$, and $\mathbb{O}^{\vee}=U_{\hbar}\left(\mathfrak{a}^{*}\right)$ is a QFSH algebra quantizing $\mathfrak{a}^{*}$.

We now compute these functors explicitly in the case of cocommutative QUE and commutative QFSH algebras. If $U=U(\mathfrak{a}) \llbracket \hbar \rrbracket$ with cocommutative coproduct (where $\mathfrak{a}$ is a Lie algebra), then $U^{\prime}$ is a completion of $U(\hbar \mathfrak{a} \llbracket \hbar \rrbracket)$; this is a flat deformation of $\hat{S}(\mathfrak{a})$ equipped with its linear Lie-Poisson structure. If $G$ is a formal group with function ring $O_{G}$, then $O:=\mathscr{O}_{G} \llbracket \hbar \rrbracket$ is a QFSH algebra, and $O^{\vee}$ is a commutative QUE algebra; it is a quantization of $S\left(\mathfrak{g}^{*}\right)$, with a commutative product, a cocommutative coproduct, and a co-Poisson structure induced by the Lie bracket of $\mathfrak{g}$.

\section{Proof that "twists" can be made admissible.}

Definition 4.1. An element $x$ in a QUE algebra $U$ is admissible if $x \in 1+\hbar U$, and if $\hbar \log x$ is in $U^{\prime} \subset U$.

In this subsection, we will prove that for $\gamma, \gamma^{\prime}$ in $\Gamma$, the twist $\mathrm{F}_{\gamma, \gamma \gamma^{\prime}}$ defined in Proposition 1.5 is twist equivalent to an admissible one.

Proposition 4.2. Let $\mathrm{F}_{\gamma, \gamma \gamma^{\prime}}$ be the element in $U^{\otimes 2}$ introduced in Proposition 1.5. Then there exists elements $\mathrm{b}_{\gamma, \gamma \gamma^{\prime}}$ in $U$ such that

$$
\mathrm{b}_{\gamma, \gamma \gamma^{\prime}}^{\mathrm{*}} \mathrm{F}_{\gamma, \gamma \gamma^{\prime}}:=\mathrm{b}_{\gamma, \gamma \gamma^{\prime}}^{\otimes 2} \mathrm{~F}_{\gamma, \gamma \gamma^{\prime}} \Delta_{\gamma}\left(\mathrm{b}_{\gamma, \gamma \gamma^{\prime}}^{-1}\right)
$$

is admissible.

Proof. Let us denote $\mathrm{F}_{0}=\mathrm{F}_{\gamma, \gamma \gamma^{\prime}}$. We will follow the proof of [Enriquez and Halbout 2007, Proposition 5.2]. Let us construct $\mathrm{b}=\mathrm{b}_{\gamma, \gamma \gamma^{\prime}}$ as a product $\cdots \mathrm{b}_{2} \mathrm{~b}_{1}$, where

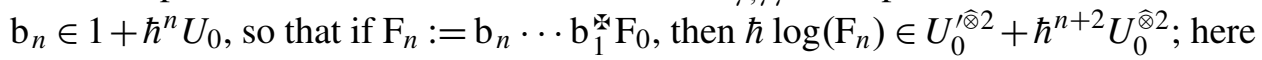
$U_{0}$ denotes the augmentation ideal. 
We have already $\hbar \log \left(\mathrm{F}_{0}\right) \in \hbar^{2} U_{0}^{\widehat{\otimes} 2}$.

Expand $\mathrm{F}_{0}=1^{\otimes 2}+\hbar \mathrm{f}_{1}+\cdots$. Then $\operatorname{Alt}\left(\mathrm{f}_{1}\right)=r$. Moreover, the coefficient of $\hbar$ in $\mathrm{F}_{0}^{1,2} \mathrm{~F}_{0}^{12,3}=\mathrm{F}_{0}^{2,3} \mathrm{~F}_{0}^{1,23}$ yields $d\left(\mathrm{f}_{1}\right)=0$, where $d: U(\mathfrak{g})_{0}^{\otimes 2} \rightarrow U(\mathfrak{g})_{0}^{\otimes 3}$ is the co-Hochschild differential. It follows that $\mathrm{f}_{1}=r+d\left(a_{1}\right)$ for some $a_{1} \in U(\mathfrak{g})_{0}$. Then if we set $\mathrm{b}_{1}:=\exp \left(\hbar a_{1}\right)$ and $\mathrm{F}_{1}=\mathrm{b}_{1}^{\otimes} \mathrm{F}_{0}$, we get $\mathrm{F}_{1} \in 1^{\otimes 2}+\hbar r+\hbar^{2} U_{0}^{\widehat{\otimes} 2}$. Then $\hbar \log \left(\mathrm{F}_{1}\right) \in \hbar^{2} r+\hbar^{3} U_{0}^{\widehat{\otimes} 2} \subset U_{0}^{\prime \widehat{\otimes} 2}+\hbar^{3} U_{0}^{\widehat{\otimes} 3}$.

Assume that for $n \geq 2$, we have constructed $\mathrm{b}_{1}, \ldots, \mathrm{b}_{n-1}$ such that

$$
\alpha_{n-1}:=\hbar \log \left(\mathrm{F}_{n-1}\right) \in U_{0}^{\prime \widehat{\otimes} 2}+\hbar^{n+1} U_{0}^{\widehat{\otimes} 2} .
$$

Let us recall two technical lemmas from [Enriquez and Halbout 2007]:

Lemma 4.3. The quotient $\left(U^{\prime}+\hbar^{n} U\right) /\left(U^{\prime}+\hbar^{n+1} U\right)$ identifies with $U(\mathfrak{g}) / U(\mathfrak{g})_{\leq n}$. In the same way, the quotient $\left(U_{0}^{(\widehat{\otimes} k}+\hbar^{n} U_{0}^{\widehat{\otimes} k}\right) /\left(U_{0}^{\prime \widehat{\otimes} k}+\hbar^{n+1} U_{0}^{\widehat{\otimes} k}\right)$ identifies with $U(\mathfrak{g})_{0}^{\otimes k} /\left(U(\mathfrak{g})_{0}^{\otimes k}\right)_{\leq n}$ and the quotient $\left(U_{0}^{\prime \widehat{\otimes} k}+\hbar^{n} U_{0}^{\widehat{\otimes} k}\right)^{\mathfrak{g}} /\left(U_{0}^{\prime \widehat{\otimes} k}+\hbar^{n+1} U_{0}^{\widehat{\otimes} k}\right)^{\mathfrak{g}}$ of $\mathfrak{g}$-invariant subspaces identifies with $\left(U(\mathfrak{g})_{0}^{\otimes k}\right)^{\mathfrak{g}} /\left(U(\mathfrak{g})_{0}^{\otimes k}\right)_{\leq n}^{\mathfrak{g}}$.

Lemma 4.4. Assume that $n \geq 2$. If $f_{1}, f_{2} \in\left(U_{0}^{\prime}\right)^{2}+\hbar^{n+1} U_{0}$ and $g, h \in \hbar^{n} U_{0}$, then $\left(f_{1}+g\right) \star_{\hbar}\left(f_{2}+h\right)=g+h$ modulo $\left(U_{0}^{\prime}\right)^{2}+\hbar^{n+1} U_{0}$, where $\star_{\hbar}$ is the $C B H$ product for the Lie bracket $[a, b]_{\hbar}=[a, b] / \hbar$.

Let us denote by $\bar{\alpha}$ the image of the class of $\alpha_{n-1}$ in $U(\mathfrak{g})_{0}^{\otimes 2} /\left(U(\mathfrak{g})_{0}^{\otimes 2}\right)_{\leq n+1}$ under the isomorphism of this space with

$$
\left(U_{0}^{\prime \widehat{\otimes} 2}+\hbar^{n+1} U_{0}^{\widehat{\otimes} 2}\right) /\left(U_{0}^{\prime \widehat{\otimes} 2}+\hbar^{n+2} U_{0}^{\widehat{\otimes} 2}\right)
$$

(see Lemma 4.3). Let $\alpha \in U(\mathfrak{g})_{0}^{\otimes 2}$ be a representative of $\bar{\alpha}$. Then $\alpha_{n-1}=\alpha^{\prime}+\hbar^{n+1} \alpha$, where $\alpha^{\prime} \in U_{0}^{\prime \widehat{\otimes} 2}+\hbar^{n+2} U_{0}^{\widehat{\otimes} 2}$. Then the twist equation gives

$\left(-\alpha^{\prime}-\hbar^{n+1} \alpha\right)^{1,23} \star_{\hbar}\left(-\alpha^{\prime}-\hbar^{n+1} \alpha\right)^{2,3} \star_{\hbar}\left(\alpha^{\prime}+\hbar^{n+1} \alpha\right)^{1,2} \star_{\hbar}\left(\alpha^{\prime}+\hbar^{n+1} \alpha\right)^{12,3}=0$.

By Lemma 4.4, the image of this equality in $\left(U^{\widehat{\otimes} 3}+\hbar^{n+1} U^{\prime \widehat{\otimes} 3}\right) /\left(U^{\widehat{\otimes} 3}+\hbar^{n+2} U^{\prime \widehat{\otimes} 3}\right) \simeq$ $U(\mathfrak{g})^{\otimes 3} /\left(U(\mathfrak{g})^{\otimes 3}\right)_{\leq n+1}$ is $d(\bar{\alpha})=0$, where $d$ is the co-Hochschild differential on the quotient $U(\mathfrak{g})_{0}^{\otimes} \cdot /\left(U(\mathfrak{g})_{0}^{\otimes} \cdot\right)_{\leq n+1}$. Since $n \geq 2$, the relevant cohomology group vanishes, so $\bar{\alpha}=d(\bar{\beta})$, where $\overline{\bar{\beta}} \in U(\mathfrak{g})_{0} /\left(U(\mathfrak{g})_{0}\right)_{\leq n+1}$. Let $\beta \in U(\mathfrak{g})_{0}$ be a representative of $\bar{\beta}$ and set

$$
\mathrm{b}_{n}:=\exp \left(\hbar^{n} \beta\right), \quad \mathrm{F}_{n}:=\mathrm{b}_{n}^{*} \mathrm{~F}_{n-1}, \quad \alpha_{n}:=\hbar \log \left(\mathrm{F}_{n}\right) .
$$

Then $\alpha_{n}=\left(\hbar^{n+1} \beta\right)^{1} \star_{\hbar}\left(\hbar^{n+1} \beta\right)^{2} \star_{\hbar} \alpha_{n-1} \star_{\hbar}\left(-\hbar^{n+1} \beta\right)^{12}$. According to Lemma 4.4, the image of $\alpha_{n}$ in

$$
\left(U_{0}^{\widehat{\otimes} 2}+\hbar^{n+1} U_{0}^{\prime \widehat{\otimes} 2}\right) /\left(U_{0}^{\widehat{\otimes} 2}+\hbar^{n+2} U_{0}^{\prime \widehat{\otimes} 2}\right) \simeq U(\mathfrak{g})_{0}^{\otimes 2} /\left(U(\mathfrak{g})_{0}^{\otimes 2}\right)_{\leq n+1}
$$

is $\bar{\alpha}-d(\bar{\beta})=0$. So $\alpha_{n}$ belongs to $U_{0}^{\widehat{\otimes} 2}+\hbar^{n+2} U_{0}^{\prime \widehat{\otimes} 2}$, as required. This proves the induction step. 
The proof of Theorem 2.5. Thanks to the previous subsection, we now know that there exists an element $\mathrm{b}_{\gamma, \gamma \gamma^{\prime}}$ in $U$ such that $\mathrm{b}_{\gamma, \gamma \gamma^{\prime}}^{\prime} \mathrm{F}_{\gamma, \gamma \gamma^{\prime}}:=\mathrm{b}_{\gamma, \gamma \gamma^{\prime}}^{\otimes 2} \mathrm{~F}_{\gamma, \gamma \gamma^{\prime}} \Delta_{\gamma}\left(\mathrm{b}_{\gamma, \gamma \gamma^{\prime}}^{-1}\right)$ is admissible. Let us define

$$
\mathrm{F}_{\gamma, \gamma \gamma^{\prime}}^{\prime}=\mathrm{b}_{\gamma, \gamma \gamma^{\prime}}^{\prime} \mathrm{F}_{\gamma, \gamma \gamma^{\prime}}, \quad \mathrm{i}_{\gamma, \gamma \gamma^{\prime}}^{\prime}=\mathrm{i}_{\gamma, \gamma \gamma^{\prime}} \circ \operatorname{Ad}\left(\mathrm{b}_{\gamma, \gamma \gamma^{\prime}}^{-1}\right)
$$

and

$$
\mathbf{v}_{\gamma, \gamma \gamma^{\prime}, \gamma \gamma^{\prime} \gamma^{\prime \prime}}^{\prime}=\mathbf{b}_{\gamma, \gamma \gamma^{\prime} \gamma^{\prime \prime}} \mathbf{v}_{\gamma, \gamma \gamma^{\prime}, \gamma \gamma^{\prime} \gamma^{\prime \prime}} \mathbf{i}_{\gamma, \gamma \gamma^{\prime}}^{-1}\left(\mathbf{b}_{\gamma \gamma^{\prime}, \gamma \gamma^{\prime} \gamma^{\prime \prime}}^{-1}\right) \mathbf{b}_{\gamma, \gamma \gamma^{\prime}}^{-1}
$$

Clearly, $\mathrm{F}_{\gamma, \gamma \gamma^{\prime}}^{\prime}, \mathrm{i}_{\gamma, \gamma \gamma^{\prime}}^{\prime}$ and $\mathrm{v}_{\gamma, \gamma \gamma^{\prime}, \gamma \gamma^{\prime} \gamma^{\prime \prime}}^{\prime}$ still satisfy the conclusion of Proposition 1.5.

Applying the functor QUE $\rightarrow$ QFSH explained on page 112 to the algebras $\left(U_{\gamma}, \gamma, \Delta_{\gamma}\right)$, we get algebras $\left(U_{\gamma}^{\prime}, *_{\gamma}, \Delta_{\gamma}\right)$, which are quantizations of the Poisson algebras $\left(\mathcal{O}_{G_{\gamma}^{*}},\{\cdot, \cdot\}_{\gamma}\right)$. Since the twists $\mathrm{F}_{\gamma, \gamma \gamma^{\prime}}^{\prime}$ are admissible, the algebra morphisms $i_{\gamma, \gamma \gamma^{\prime}}^{\prime}$ restrict to the QFSH algebras $U_{\gamma}^{\prime}$. Then Theorem 2.5 will follow from this:

Proposition 4.5. The elements $\mathrm{v}_{\gamma, \gamma \gamma^{\prime}, \gamma \gamma^{\prime} \gamma^{\prime \prime}}^{\prime}$ are admissible.

Proof. Let us denote $\mathrm{v}=\mathrm{v}_{\gamma, \gamma \gamma^{\prime}, \gamma \gamma^{\prime} \gamma^{\prime \prime}}^{\prime}$. Suppose $\mathrm{v}$ is not admissible and let $n$ be the bigger $i$ such that $\alpha_{0}:=\hbar \log (\mathrm{v}) \in U_{0}+\hbar^{n+1} U_{0}$. By the assumption on $v$, we know that $n \geq 2$. Let us denote by $\bar{\alpha}$ the image of the class of $\alpha_{0}$ in $U(\mathfrak{g})_{0} /\left(U(\mathfrak{g})_{0}\right)_{\leq n+1}$ under the isomorphism of this space with $\left(U_{0}+\hbar^{n+1} U_{0}\right) /\left(U_{0}+\hbar^{n+2} U_{0}\right)$; see Lemma 4.3. Let $\alpha \in U(\mathfrak{g})_{0}$ be a representative of $\bar{\alpha}$. Then $\alpha_{0}=\alpha^{\prime}+\hbar^{n+1} \alpha$, where $\alpha^{\prime} \in U_{0}+\hbar^{n+2} U_{0}$. Let $f, f^{\prime}$ and $f^{\prime \prime}$ be respectively the $\hbar \operatorname{logs}$ of $\mathrm{F}_{\gamma, \gamma \gamma^{\prime}}^{\prime}, \mathrm{F}_{\gamma \gamma^{\prime}, \gamma \gamma^{\prime} \gamma^{\prime \prime}}^{\prime}$ and $\mathrm{F}_{\gamma, \gamma \gamma^{\prime} \gamma^{\prime \prime}}$. Then the compatibility equation for composition of twists gives

$$
f^{\prime \prime}=\left(\alpha^{\prime}+\hbar^{n+1} \alpha\right)^{\otimes 2} \star_{\hbar} \mathrm{i}_{\gamma, \gamma \gamma^{\prime}}^{-1}\left(f^{\prime}\right) \star_{\hbar} f \star_{\hbar}\left(-\alpha^{\prime}-\hbar^{n+1} \alpha\right)^{12}=0 .
$$

According to Lemma 4.4, the image of this equation in

$$
\left(U^{\widehat{\otimes} 2}+\hbar^{n+1} U^{\prime \widehat{\otimes} 2}\right) /\left(U^{\widehat{\otimes} 2}+\hbar^{n+2} U^{\prime \widehat{\otimes} 2}\right) \simeq U(\mathfrak{g})^{\otimes 2} /\left(U(\mathfrak{g})^{\otimes 2}\right)_{\leq n+1}
$$

is $d(\bar{\alpha})=0$. So $\bar{\alpha} \in \mathfrak{g}$, which is a contradiction with $n \geq 2$.

\section{Example of simple group with action of the Weyl group}

Quantization of Majid and Soubel'man. We start by briefly recalling Majid and Solbel'man's approach [1994] to the quantum Weyl group. Let $\mathfrak{g}$ be a complex simple Lie algebra, and let $U_{\hbar}(\mathfrak{g})$ be the natural deformation of the universal enveloping algebra $U(\mathfrak{g})$. Lustig [1990] and Sořbel'man [1991] first independently noticed that a simple reflection $w$ in the Weyl group $W$ of $\mathfrak{g}$ defines an automorphism $\alpha_{w}$ on $U_{\hbar}(\mathfrak{g})$. Then one can extend $U_{\hbar}(\mathfrak{g})$ by elements $\bar{w}$ with $\alpha_{w}(g)=\bar{w} g \bar{w}^{-1}$ for all simple reflections in $W$. The extended algebra is called the "quantum Weyl group" and denoted by $\widehat{U_{\hbar}(\mathfrak{g})}$. In [Kirillov and Reshetikhin 1990] and [Sor̆bel'man 1991], this algebra is used to construct explicit solutions to the Yang-Baxter equation. 
Majid and Sorbel'man also discovered the bicrossed product structure on $\widetilde{U_{\hbar}(\mathfrak{g}) \text {. }}$ For $1 \leq i, j \leq \operatorname{rank}(\mathfrak{g})$, let $w_{i}$ be simple reflections in $W$, and let $t_{j}$ be elements in the maximal torus corresponding to $\phi_{j}\left(\begin{array}{rr}-1 & 0 \\ 0 & -1\end{array}\right)$ with $\phi_{j}: s l_{2} \hookrightarrow \mathfrak{g}$ embedding to the $j$-th vertex of the Dynkin diagram. Then define $\widetilde{W}$ to be the group generated by $w_{i}$ and $t_{j}$, which is a covering of the Weyl group $W$ with the kernel isomorphic to the direct sum of $k$-copies of $\mathbb{Z}_{2}$, where $k=\operatorname{rank}(\mathfrak{g})$. The quantum Weyl group $\widehat{U_{\hbar}(\mathfrak{g})}$ is proved in [Majid and Soĭbel'man 1994, Corollary 3.4] to be isomorphic to the bicrossed product

$$
k \widetilde{W}^{\psi} \bowtie_{\alpha, \chi} U_{\hbar}(\mathfrak{g}),
$$

defined in terms of linear maps

$$
\begin{array}{ll}
\alpha: U_{\hbar}(\mathfrak{g}) \otimes k \widetilde{W} \rightarrow U_{q}(g), & a \otimes w t \mapsto t^{-1} \alpha_{w}(a) t, \\
\chi: k \widetilde{W} \otimes k \widetilde{W} \rightarrow U_{\hbar}(\mathfrak{g}), & w_{1} t_{1} \otimes w_{2} t_{2} \mapsto x^{-1}, \\
\psi: k \widetilde{W} \rightarrow U_{\hbar}(\mathfrak{g}) \otimes U_{\hbar}(\mathfrak{g}), & w t \mapsto\left(\bar{w}^{-1} \otimes \bar{w}^{-1}\right) \Delta \bar{w} .
\end{array}
$$

Here, $x$ is an element in $U_{\hbar}(\mathfrak{g})$ such that $\alpha_{w_{1} w_{2}\left(\alpha_{w_{1}}\left(t_{1}\right) t_{2}\right)}=\alpha_{w_{1} t_{1}} \alpha_{w_{2} t_{2}} \operatorname{Ad}_{x^{-1}}$ with $x \in U_{\hbar}(\mathfrak{g})$.

Proposition 5.1. The quantum Weyl group $\widehat{U_{\hbar}(\mathfrak{g})}$ is a quantization of the $\Gamma=\widetilde{W}$ Lie bialgebra $(\mathfrak{g},[\cdot, \cdot], \delta)$, where $(\mathfrak{g},[\cdot, \cdot], \delta)$ is the Lie bialgebra structure on $\mathfrak{g}$ corresponding to the deformation $U_{\hbar}(\mathfrak{g})$, and $\widetilde{W}$ acts on $\mathfrak{g}$ as the Weyl group ( $t$ acts on $\mathfrak{g}$ by adjoint action), and $f_{\gamma}=\bigwedge^{2}(\gamma) \circ \delta \circ \gamma^{-1}-\delta$ for $\gamma \in \widetilde{W}$.

Proof. Inspired by the above bicrossed product structure on $\widetilde{U_{\hbar}(\mathfrak{g})}$, we introduce the $\Gamma$ quantized universal enveloping algebras for $\Gamma=\widetilde{W}$ generated as follows:

- Set $\left(U_{\hbar}(\mathfrak{g})_{\gamma}, m_{\gamma}, \Delta_{\gamma}\right)=\left(U_{\hbar}(\mathfrak{g}), m, \Delta_{\gamma}\right)$, where $m$ is the canonical multiplication on $U_{\hbar}(\mathfrak{g})$ and $\Delta_{\gamma}=\alpha(\cdot, \gamma)^{\otimes 2} \circ \operatorname{Ad}(\psi(\gamma)) \circ \Delta \circ \alpha^{-1}(\cdot, \gamma)$ with $\Delta$ the canonical coproduct on $U_{\hbar}(\mathfrak{g})$.

- Define $i_{\gamma, \gamma \gamma^{\prime}}:\left(U_{\hbar}(\mathfrak{g}), m_{\gamma}\right) \rightarrow\left(U_{\hbar}(\mathfrak{g}), m_{\gamma \gamma^{\prime}}\right)$ by $i_{e, \gamma}=\alpha(\cdot \otimes \gamma): U_{\hbar}(\mathfrak{g}) \rightarrow U_{\hbar}(\mathfrak{g})$ and $i_{\gamma, \gamma \gamma^{\prime}}=i_{e, \gamma^{\prime}}$.

- Set $F_{e, \gamma} \in U_{\hbar}(\mathfrak{g})^{\otimes 2}$ equal to $\psi(\gamma)$ and put $F_{\gamma, \gamma \gamma^{\prime}}=F_{e, \gamma^{\prime}}$. By [Majid and Soĭbel'man 1994, Lemma 3.3], we have

$$
F_{e, w_{i} t}=\psi\left(w_{i}\right)=e^{\frac{1}{2} \hbar H_{i} \otimes H_{i} /\left(\alpha_{i}, \alpha_{i}\right)}\left(\mathscr{R}_{i}\right)_{12}^{-1}=1+\hbar f_{1}+O\left(\hbar^{2}\right)
$$

for any reflection $w_{i} \in W$. (Here $\left(H_{i}, X_{i}^{+}, X_{i}^{-}\right)$corresponds to the embedding $\phi_{i}: s l_{2} \hookrightarrow \mathfrak{g}$ for the $i$-th root $\alpha_{i}$ with normal $\left(\alpha_{i}, \alpha_{i}\right)$.) Because the first order part of $e^{\frac{1}{2} \hbar H_{i} \otimes H_{i} /\left(\alpha_{i}, \alpha_{i}\right)}$ is symmetric, the antisymmetrization of $f_{1}$ is equal to the antisymmetrization of the first order term of $\left(\mathscr{R}_{i}\right)_{21}^{-1}$, which is equal to the definition of $f_{w_{i}}$ by the asymptotic expansion of $\mathscr{R}_{i}$. This result extends to an arbitrary element $\gamma$ simply because $w_{i}$ generates $W$. 
- Set $v_{e, \gamma, \gamma \gamma^{\prime}}=\chi\left(\gamma, \gamma \gamma^{\prime}\right) \in U_{\hbar}(\mathfrak{g})^{\otimes 2}$. By the definition of $\chi\left(\gamma, \gamma \gamma^{\prime}\right)$, we can choose $v$ to be an element in $1+\hbar^{2} U_{\hbar}(\mathfrak{g})$ because the $\alpha$ action is associative up to the $\hbar$-linear terms by [Kirillov and Reshetikhin 1990, Formula (13)] and [Levendorskiı̌ and Sořbel'man 1990, Prop 1.4.10].

It is easy to check that the cocycle conditions for $\alpha, \chi, \psi$, and their compatibilities are equivalent to the conditions for $\left(U_{\hbar}, m, \Delta_{\gamma}, i_{\gamma, \gamma \gamma^{\prime}}, F_{\gamma, \gamma \gamma}, v_{\gamma, \gamma \gamma^{\prime}, \gamma \gamma^{\prime} \gamma^{\prime \prime}}\right)$ to be a $\Gamma=\widetilde{W}$ quantized universal enveloping algebra. Therefore, the corresponding $\Gamma$ quantized universal enveloping algebra is isomorphic to $\widehat{U_{\hbar}(\mathfrak{g})}$.

\section{Admissibility of the twists.}

Corollary 5.2. The twists $F_{\gamma, \gamma \gamma^{\prime}}$ and $v_{\gamma, \gamma \gamma^{\prime}, \gamma \gamma^{\prime} \gamma^{\prime \prime}}$ defined in Proposition 5.1 are admissible. Therefore, the quantum Weyl group defines a stack of formal series Hopf algebras quantizing the corresponding stack of Poisson-Hopf algebras dual to $\left(\widetilde{W}, \mathfrak{g},[\cdot, \cdot], \delta, f_{\gamma}\right)$.

Proof. We look at the formulas for $F_{e, w t}$. By the one for $\psi$, if $w_{i}$ is a simple reflection, then $F_{e, w_{i} t}=e^{\frac{1}{2} \hbar H_{i} \otimes H_{i} /\left(\alpha_{i}, \alpha_{i}\right)}\left(\mathscr{R}_{i}\right)_{12}^{-1}$. Taking $\hbar \log$ on $F_{e, w}$, we have

$$
\hbar^{2} \frac{1}{2} H_{i} \otimes H_{i} /\left(\alpha_{i}, \alpha_{i}\right)+\hbar \log \left(\left(\Re_{i}\right)_{12}^{-1}\right) .
$$

The first term is primitive as $H_{i}$ is primitive, and the second term $\hbar \log \left(\left(\mathscr{R}_{i}\right)_{12}^{-1}\right)$ is primitive because $\hbar \log \left(\mathscr{R}_{i}\right)$ is primitive, which was proved in [Enriquez and Halbout 2003, Theorem 0.1]. Therefore, we conclude that $F_{e, w_{i} t}$ is admissible when $w$ is a simple reflection. This property extends to a general element $\gamma$ directly by products.

By Proposition 4.5, we also know that $v$ is admissible because $F$ is admissible.

We conclude the corollary by Theorem 2.5 .

\section{Acknowledgment}

We thank Benjamin Enriquez for very useful discussions.

\section{References}

[Bressler et al. 2007] P. Bressler, A. Gorokhovsky, R. Nest, and B. Tsygan, "Deformation quantization of gerbes”, Adv. Math. 214:1 (2007), 230-266. MR 2008m:53210 Zbl 1125.53069

[Drinfeld 1987] V. G. Drinfel'd, “Quantum groups”, pp. 798-820 in Proceedings of the International Congress of Mathematicians (Berkeley, 1986), vol. 1, edited by A. M. Gleason, Amer. Math. Soc., Providence, RI, 1987. MR 89f:17017

[Drinfeld 1989] V. G. Drinfel'd, "Quasi-Hopf algebras", Algebra i Analiz 1:6 (1989), 114-148. In Russian; translated in Leningrad Math. J. 1:6 (1990), 1419-1457. MR 91b:17016 Zbl 0718.16033

[Enriquez and Halbout 2003] B. Enriquez and G. Halbout, "An $\hbar$-adic valuation property of universal R-matrices", J. Algebra 261:2 (2003), 434-447. MR 2004a:17015 Zbl 1015.17008 
[Enriquez and Halbout 2007] B. Enriquez and G. Halbout, "Coboundary Lie bialgebras and commutative subalgebras of universal enveloping algebras", Pacific J. Math. 229:1 (2007), 161-184. MR 2007m:17028 Zbl 05366189

[Enriquez and Halbout 2008] B. Enriquez and G. Halbout, "Quantization of $\Gamma$-Lie bialgebras", $J$. Algebra 319:9 (2008), 3752-3769. MR 2009b:17050 Zbl 1144.17016

[Enriquez et al. 2003] B. Enriquez, F. Gavarini, and G. Halbout, "Uniqueness of braidings of quasitriangular Lie bialgebras and lifts of classical $r$-matrices", Int. Math. Res. Not. 2003:46 (2003), 2461-2486. MR 2004m:17045 Zbl 1044.17019

[Gavarini 2002] F. Gavarini, "The quantum duality principle", Ann. Inst. Fourier (Grenoble) 52:3 (2002), 809-834. MR 2003d:17016 Zbl 1054.17011

[Kirillov and Reshetikhin 1990] A. N. Kirillov and N. Reshetikhin, " $q$-Weyl group and a multiplicative formula for universal $R$-matrices”, Comm. Math. Phys. 134:2 (1990), 421-431. MR 92c:17023 Zbl 0723.17014

[Levendorskiı̌ and Soŭbel'man 1990] S. Z. Levendorskiĭ and Y. S. Sořbel'man, "Some applications of the quantum Weyl groups”, J. Geom. Phys. 7:2 (1990), 241-254. MR 92g:17016 Zbl 0729.17009

[Lusztig 1990] G. Lusztig, "Quantum groups at roots of 1”, Geom. Dedicata 35:1-3 (1990), 89-113. MR 91j:17018 Zbl 0714.17013

[Majid and Soĭbel'man 1994] S. Majid and Y. S. Sořbel'man, "Bicrossproduct structure of the quantum Weyl group”, J. Algebra 163:1 (1994), 68-87. MR 95a:17020 Zbl 0801.17014

[Sořbel'man 1991] Y. S. Sořbel'man, "Quantum Weyl group and some [of] its applications”, pp. 233-235 in Proceedings of the Winter School on Geometry and Physics (Srní, 1990), edited by J. Bureš and V. Souček, 1991. MR 93d:17022 Zbl 0753.17029

Received October 27, 2008. Revised August 13, 2009.

\title{
Gilles Halbout
}

Institut de Mathématiques et De Modélisation De MontPellier

UNIVERSITÉ DE MONTPELLIER 2

CC5149, PlaCe EugÈne Bataillon

F-34095 MONTPELLIER CEDEX 5

FRANCE

halbout@math.univ-montp2.fr

\author{
XiAng TANG \\ DEPARTMENT OF MATHEMATICS \\ WASHINGTON UNIVERSITY \\ ST. LOUIS, MO 63130 \\ UNITED STATES \\ xtang@math.wustl.edu
}

